\title{
Quantitative MR Markers in Non-myelopathic Spinal Cord Compression: A Narrative review
}

\author{
Jan Valošek 1,2,3, Petr Bednařík 4,5, Miloš Keřkovský 6,7, Petr Hluštík 1,10, Josef Bednařík 6,8,9 and Alena Svátková 11,*
}

1 Department of Neurology, Faculty of Medicine and Dentistry, Palacký University Olomouc, Olomouc, Czechia; jan.valosek@upol.cz (J.V.); phlustik@upol.cz (P.H.)

2 Department of Radiology, Faculty of Medicine and Dentistry, Palacký University Olomouc, Olomouc, Czechia

3 Department of Biomedical Engineering, University Hospital Olomouc, Olomouc, Czechia

4 Danish Research Centre for Magnetic Resonance, Centre for Functional and Diagnostic Imaging and Department of Radiology, Centre for Functional and Diagnostic Imaging and Research, Copenhagen University Hospital Amager and Hvidovre, Hvidovre Denmark; petrb@drcmr.dk (P.B.)

5 Department of Radiology, Centre for Functional and Diagnostic Imaging and Research, Copenhagen University Hospital Amager and Hvidovre, Hvidovre, Denmark

6 Faculty of Medicine, Masaryk University, Brno, Czechia; Kerkovsky.Milos@fnbrno.cz (M.K.); Benarik.Josef@fnbrno.cz (J.B.)

7 Department of Radiology and Nuclear Medicine, University Hospital Brno, Brno, Czechia

8 Department of Neurology, University Hospital Brno, Brno, Czechia

9 Central European Institute of Technology, Masaryk University, Brno, Czechia

10 Department of Neurology, University Hospital Olomouc, Olomouc, Czechia

11 Department of Medicine III, Clinical Division of Endocrinology and Metabolism, Medical University of Vienna, Vienna, Austria alena.svatkova@meduniwien.ac.at (A.S.)

* Correspondence: alena.svatkova@meduniwien.ac.at (A.S.)

\begin{abstract}
Degenerative spinal cord (SC) compression is a frequent pathological condition with increasing prevalence throughout aging. Initial non-myelopathic cervical SC compression (NMDC) might progress over time into potentially irreversible degenerative cervical myelopathy (DCM). While quantitative MRI (qMRI) techniques demonstrated the ability to depict intrinsic tissue properties, longitudinal in-vivo biomarkers to identify NMDC patients who will eventually develop DCM are still missing. Thus, we aim to review the ability of qMRI techniques (such as diffusion MRI, diffusion tensor imaging (DTI), magnetization transfer (MT) imaging, magnetic resonance spectroscopy ( $\left.\left.{ }^{1} \mathrm{H}-\mathrm{MRS}\right)\right)$ to serve as prognostic markers in NMDC. While DTI in NMDC patients consistently detected lower fractional anisotropy and higher mean diffusivity at compressed levels caused by demyelination and axonal injury, MT and ${ }^{1} \mathrm{H}-\mathrm{MRS}$ along with advanced and tract-specific diffusion MRI recently revealed microstructural alterations also rostrally pointing to Wallerian degeneration. Recent studies also disclosed significant relationship between microstructural damage and functional deficits, assessed by qMRI and electrophysiology, respectively. Thus, tract-specific qMRI in combination with electrophysiology critically extend our understanding of the underlying pathophysiology of degenerative SC compression and may provide predictive markers of DCM development for accurate patient management. However, the prognostic value must be validated in longitudinal studies.
\end{abstract}

Keywords: non-myelopathic cervical spinal cord compression; degenerative cervical myelopathy; diffusion magnetic resonance imaging; quantitative magnetic resonance imaging

\section{Introduction}

Degenerative spinal cord (SC) compression frequently occurs in the elderly due to pathological changes such as intervertebral disc bulging, herniation or osteophyte formation throughout aging [1-4]. Relative resilience of the cervical SC to incipient 
compressive changes often leads to non-myelopathic degenerative cervical spinal cord compression (NMDC), a condition that precedes clinically manifest degenerative cervical myelopathy (DCM) [4-7]. Although the cervical SC compression occurs predominantly between $C 4 / 5$ and C6/7 cervical levels $[2,8]$, secondary degenerative changes such as axonal degeneration and demyelination propagate remotely in both superior and inferior directions affecting SC levels above and below the compression and even leading to changes in the brain [9-11]. The recent systematic review [1] showed that the prevalence of NMDC in Caucasian population over 60 years is up to $39.7 \%$ and further increases with age $[1,2,12]$. Although NMDC patients may only exhibit cervical axial pain and/or signs or symptoms of radiculopathy (Table 1) without any signs or symptoms of clinical manifest myelopathy, ${ }^{1}$ up to $23 \%$ of NMDC patients progress into symptomatic DCM during a follow-up of 44 months [6,13]. The current clinical guidelines [7] and Recommendations of World Federation of Neurosurgical Societies Spine Committee [14] imply conservative clinical treatment in NMDC patients without symptoms of radiculopathy, whereas recommending a consideration of surgical intervention in NMDC patients with clinical and/or electrophysiological evidence of radiculopathy. However, the surgery is associated with risks of neurological deterioration in $7-11 \%$ of patients after surgery [4]. Given the undeniable risks of decompressive surgery, the aging of the population worldwide, and substantially reduced quality of life in DCM patients [4,15], there is an urgent need to reliably identify NMDC patients with a higher risk of progression to irreversible DCM [14].

Quantitative magnetic resonance imaging (qMRI) techniques such as diffusion MRI $(\mathrm{dMRI})$, diffusion tensor imaging (DTI), ${ }^{2}$ magnetization transfer (MT) imaging, or singlevoxel magnetic resonance spectroscopy ( $\left.{ }^{1} \mathrm{H}-\mathrm{MRS}\right)$ allow assessing SC microstructure and provide crucial in-vivo insight into pathophysiology of degenerative compression, which is not accessible by conventional clinical MRI [16-18].

While recent reviews [4,9,19-22] focused on epidemiology, pathophysiology, and assessment of DCM using structural MRI, DTI [23,24] and MRS [24], so far, limited attention has been paid to the NMDC patients. To date, a single systematic review by Smith et al. [1] covered the NMDC prevalence in structural MRI but did not discuss the benefits and pitfalls of qMRI techniques in NMDC. Thus, our review aims to identify and discuss the potential of novel qMRI techniques to quantify NMDC alterations in vivo and determine the likeliness of progression to DCM. Due to the relatively limited number of NMDC studies using qMRI techniques, DCM studies were also included to elaborate their prospects in NMDC. We also summarized innovative emerging MRI techniques for the assessment of SC compression.

1 Objective physical signs of myelopathy include upper motor neuron signs in the upper and/or lower limbs (for example, hyper-reflexia, clonus, a positive Hoffman sign, a positive Trömner sign, an upgoing plantar response and lower limb spasticity), corticospinal tract distribution motor deficits, atrophy of intrinsic hand muscles, dermatomal sensory loss and a broad-based, unstable gait [4].

2 Many studies refer diffusion tensor imaging (DTI) as a separate method or technique, but in fact it is only the simplest model used for estimation of diffusion directionally from diffusion MRI (dMRI/DWI) [49]. 
Table 1. Nomenclature and definitions of non-myelopathic spinal cord compression across studies.

\begin{tabular}{c}
\hline Study \\
Bednarik et al. 2004 [5], 2008 [6] \\
Keřkovský et al. 2012 [25]
\end{tabular}

\section{Nomenclature \\ Original articles}

Pre-symptomatic spondylotic cervical cord compression (P-

SCCC)

Adamova et al. 2015 [3]

Kovalova et al. 2016 [2]

Keřkovský et al. 2017 [26]

Ellingson et al. 2018 [27]

Martin et al. 2018 [28]

Asymptomatic spondylotic cervical cord compression (ASCCC)

Nonmyelopathic spondylotic cervical cord compression (NMSCCC)

Asymptomatic spondylotic cer- MR signs of spondylotic cervical vical cord encroachment (SCCE) SC compression and cervical pain and/or symptoms/signs of cervical radiculopathy, but without symptoms/signs of cervical spondylotic myelopathy

$$
(\mathrm{mJOA}=18)
$$

no detailed description

MR signs of cervical SC compression and no myelopathic signs but possible presence of radiculopathy (mJOA not reported)

Asymptomatic degenerative cer- MR finding of SC compression vical cord compression (ADCCC) and various clinical signs of cervical spine degenerative disease (cervical pain, radiculopathy) but no signs or symptoms of

$$
\mathrm{DCM}(\mathrm{mJOA}=18)
$$

Asymptomatic cervical stenosis no neurological symptomatolpatients ogy $(\mathrm{mJOA}=18)$ but complaints of neck pain

Asymptomatic spinal cord com- MR finding of cervical SC compression (ASCC) pression but an absence of any neurological symptoms and signs; neck pain was not considered a neurological symptom $(\mathrm{mJOA}=18)$

Kadanka Jr. et al. 2017 [29], La- Nonmyelopathic degenerative bounek et al. 2020 [30] cervical cord compression

(NMDCCC)

Kadanka Jr. et al. 2021 [31] Non-myelopathic degenerative cervical cord compression

(NMDCC)
MR signs of SC compression but an absence of any myelopathic signs or possible presence of axial pain and/or symptoms or signs of upper extremity monoradiculopathy or completely asymptomatic individuals (mJOA not reported) MR signs of cervical SC compression and presence of maximally one clinical myelopathic symptom but no clinical myelopathic signs $(\mathrm{mJOA} \geq 17$ ) 


\begin{tabular}{|c|c|c|}
\hline $\begin{array}{c}\text { Valošek et al. } 2021 \text { [8], Horak et } \\
\text { al. } 2021 \text { [32], Horakova et al. } \\
2022 \text { [33] }\end{array}$ & $\begin{array}{l}\text { Non-myelopathic degenerative } \\
\text { cervical spinal cord compression } \\
\text { (NMDC) }\end{array}$ & $\begin{array}{l}\text { MR signs of cervical SC com- } \\
\text { apression with or without radicu- } \\
\text { lopathy and electrophysiological } \\
\text { changes but without myelopa- } \\
\text { thic signs (mJOA }=18 \text { ) }\end{array}$ \\
\hline \multicolumn{3}{|c|}{ Reviews } \\
\hline Wilson et al. 2013 [13] & $\begin{array}{l}\text { Nonmyelopathic patients with } \\
\text { cervical stenosis }\end{array}$ & review - no single definition \\
\hline Witiw et al. 2018 [12] & $\begin{array}{l}\text { Asymptomatic cervical spinal } \\
\text { cord compression (CSCC) }\end{array}$ & review - no single definition \\
\hline Smith et al. 2020 [1] & $\begin{array}{c}\text { Asymptomatic spinal cord com- } \\
\text { pression (ASCC) }\end{array}$ & review - no single definition \\
\hline Badhiwala et al. 2020 [4] & $\begin{array}{l}\text { Cervical spinal cord compres- } \\
\text { sion without myelopathy }\end{array}$ & $\begin{array}{l}\text { review - MR signs of cervical SC } \\
\text { compression, absence of any } \\
\text { myelopathic signs and clinical } \\
\text { radiculopathy with or without } \\
\text { electrophysiological changes or } \\
\text { no signs of symptoms of radicu- } \\
\text { lopathy }(\mathrm{mJOA}=18)\end{array}$ \\
\hline
\end{tabular}

mJOA - modified Japanese Orthopaedic Association scale, SC - spinal cord

\section{MRI in the non-myelopathic and myelopathic spinal cord compression}

\subsection{Structural MRI}

Structural or conventional MRI including T1-, T2-, T2*-weighted (T1-w, T2-w, $\mathrm{T} 2 *-\mathrm{W})$, and short tau inversion recovery images in clinical routine depict macrostructural information about the SC tissue and evaluate the severity of compression and SC atrophy.

Conventional clinical MRI is primarily acquired in sagittal orientation to evaluate signal abnormalities of SC, such as the presence of T2-w hyperintensities and T1-w hypointensities [34]. Subjectively-evaluated T2-w hyperintensities are still considered an important factor influencing decision-making for decompressive surgery [13], although their presences does not necessarily correspond with the clinical DCM signs and symptoms [35]. Intramedullary T2-w hyperintensities have been indeed reported in 58-85\% of patients with clinically manifest DCM [36], whereas in NMDC cohorts inconsistently ranging between $2.3 \%-24.6 \%[2,6,29,37]$. T1-w hypointensities are associated with permanent SC injury [34] and are relatively rare, occurring in 19-30\% of DCM patients [35]; thus they predictive value in NMDC patients is limited.

In addition to the conventional clinical description of signal changes, T1-W, T2-w and $\mathrm{T} 2{ }^{*}$-w images with sufficient axial in-plane resolution below $1 \mathrm{~mm}$ and good contrast between white/gray matter (WM/GM) and cerebrospinal fluid (CSF) (typically 3D isotropic T1-w and 2D axial multi-echo gradient echo sequences) allow assessing morphometric metrics to further validate the severity of compression. Indeed, the degree of SC compression at maximally compressed level (MCL) assessed by the cross-sectional area (i.e., area of the SC in axial plane) and the compression ratio (i.e., ratio between the anteroposterior diameter and the transverse diameter) were reported as promising predictors of DCM development (Figure 1A) [2,4,6,29]. In addition, newly-proposed metrics reflecting SC flattening, indentation and torsion [33,38] semi-automatically detected SC compression with lower variability than manual raters (Figure 1A) [33] and T2*-w WM/GM ratio together with fractional anisotropy and magnetization transfer ratio discriminated between NMDC patients and healthy controls (HC) [28]. SC volumetry adds to the compression metrics at MCL when assessing changes above and below the compression levels. So far, studies demonstrated gradual reduction of SC, WM and GM volumes at C2/3 above the compression level in DCM and NMDC relative to HC and each other (Figure 2) [8,32,39- 
43], pointing to more severe Wallerian neurodegeneration and atrophy in DCM than NMDC [8,41] with similar changes in DCM also caudally at T11-L1 level [43]. However, morphometric and volumetric metrics might suffer from inter-subject variability due to anatomical and biological factors such as age, sex, height or weight, which showed significant correlations in healthy subjects and patients with SC compression [2,44-46]. Thus, qMRI metric normalization or statistical models adjusted for these factors are commonly used $[2,28,45,46]$. Also, proper SC and WM/GM segmentations for reliable metrics and volumes extraction require robust automated segmentation methods that are complemented by results quality control and potential manual correction, which is particularly needed with patients with severe compression (see Chapter 2.3.2).

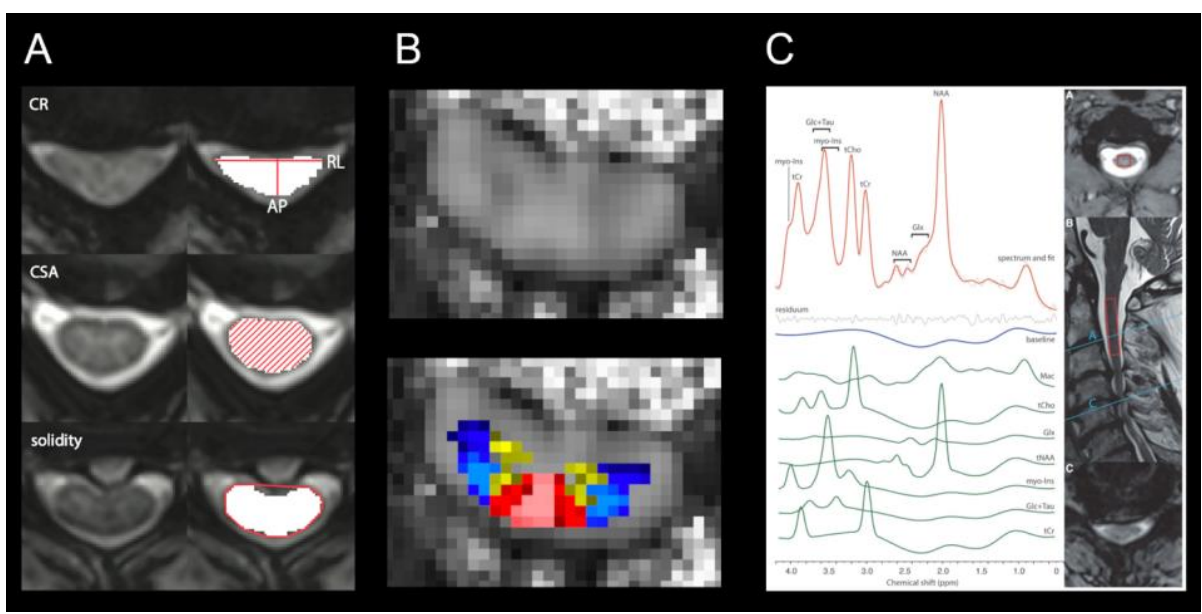

Figure 1. Quantitative MRI (qMRI) markers derived using various qMRI methods. (a) Morphometric metrics measuring the degree of spinal cord compression based on structural MRI. CR - compression ratio calculated as a ratio between the anteroposterior (AP) diameter and the transverse (RL) diameter, CSA - cross-sectional area, Solidity - calculated as a ratio of CSA to the area of the smallest convex polygon surrounding all positive pixels in the image. Adapted from [33], CC BYNC (https://creativecommons.org/licenses/by-nc/4.0/); (b) Map of fractional anisotropy (FA) estimated using diffusion tensor imaging model from diffusion-weighted imaging data. The upper panel shows FA map, lower panel shows FA map overlayed with probabilistic PAM50 atlas [47] of white and gray matter allowing tissue-specific analysis. Adapted from [8], CC BY-NC; (c) Singlevoxel magnetic resonance spectroscopy ( $\left.{ }^{1} \mathrm{H}-\mathrm{MRS}\right)$ measuring metabolic concentrations from above the compression level C2/3 (red box). Adapted from [32], CC BY-NC.

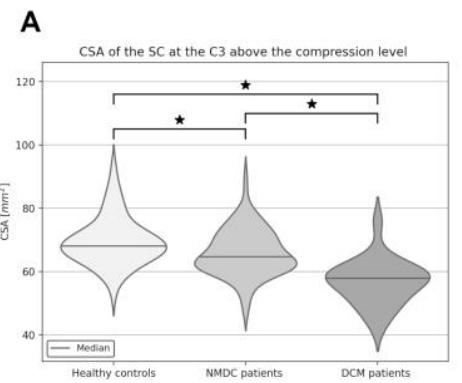

B

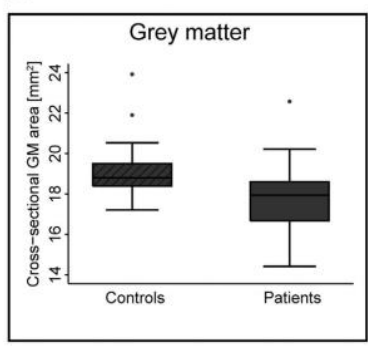

C

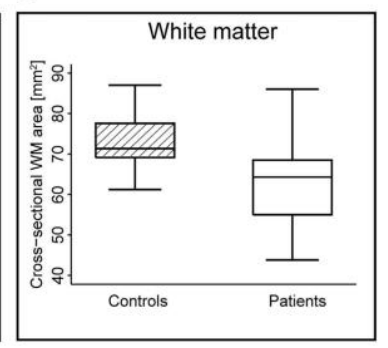

Figure 2. Significant reduction of the cross-sectional area (CSA) above the stenosis level. (a) Spinal cord (SC) CSA reduction at C3 level between NMDC and DCM patients relative to healthy controls (HC). Adapted from [8], CC BY-NC (https://creativecommons.org/licenses/by-nc/4.0/); (b) Grey and (c) white CSA reduction at C2/3 level between DCM patients and HC. Adapted from [40], CC-BY-NC. 


\subsection{Microstructural quantitative MRI}

\subsubsection{Diffusion MRI}

Diffusion magnetic resonance imaging (dMRI, or diffusion-weighted imaging, DWI) is sensitive to random water molecule movement within the tissue [48]. The tissue architecture, such as level of myelination and axonal configuration restricts the diffusion and results in measurable signal attenuation, which can be mathematically reconstructed using various models [48]. Whereas in the clinical routine, dMRI has been used for quantification of diffusion restriction or apparent diffusion coefficient, the research applications usually rely on fitting of diffusion models. The most commonly used diffusion model in the SC research is diffusion tensor imaging (DTI) $[9,16,49]$, which estimates a single tensor in each voxel. DTI provides several quantitative markers sensitive to various microstructural pathologies such as fractional anisotropy (FA) (Figure 1B), referring to the directional preference of diffusion affected by the degree of myelination, axonal packing, axon size, and/or coherence and co-linearity of fiber organization [48]. Whereas FA ranges from values close to 0 in the tissues with no boundaries for water movement (e.g., CSF) to values around 1 in highly anisotropic tissues with parallel and highly organized fiber structure, mean diffusivity (MD) measures the overall molecular diffusion rate. Axial (AD) and radial diffusivity (RD) then provide diffusion rates in the main and transverse axes, referring to the degree of tissue edema, axonal damage, and demyelination, respectively $[17,48]$. However, DTI as a single-compartment model allows to reconstruct only a primary diffusion direction and fails to estimate more complex WM fiber configurations like crossing or bending fibers [49]. Higher-order diffusion models such as neurite orientation dispersion and density imaging (NODDI) [50-55], Ball-and-Sticks [8,30], diffusion kurtosis imaging (DKI) [56,57], AxCaliber [58,59], composite hindered and restricted model of diffusion (CHARMED) [60], Q-space imaging (QSI) [57,61,62] or q-ball imaging [63], which overcome DTI's limitation by modeling several tissue compartments were recently translated from the brain to SC imaging to provide a more precise depiction of complex SC microstructure.

\subsubsection{Diffusion tensor imaging}

Multiple studies [23,25,26,30,36,38,42,55,64-80] and reviews [23,24] covered DTI in symptomatic DCM patients, whereas a limited number of works focused on NMDC patients [8,25-30] (Table 2). So far, NMDC studies have also suffered from a lack of nomenclature and inclusion criteria consistency that differs between regions and countries (Table 1). Some studies indeed included only NMDC subjects without any symptoms [28], while others also incorporated those with radiculopathy $[8,25,26]$. One of the first $1.5 \mathrm{~T}$ studies in NMDC patients compared DTI metrics from a single region of interest (ROI) from the entire axial SC of $13 \mathrm{HC}$ with 20 DCM patients (mJOA < 18) and 32 NMDC patients with cervical pain and/or radiculopathy without symptoms/signs of myelopathy $(\mathrm{mJOA}=18)$ and detected lower FA and higher MD at MCL in DCM patients compared to NMDC patients, with lower FA and no significant MD deficits between NMDC patients and HC [25]. Conclusions between NMDC and DCM patients were confirmed in a second study [26] on a group of 37 DCM patients, 93 NMDC patients and $71 \mathrm{HC}$ with the same inclusion/exclusion criteria, although no comparison between NMDC patients and HC was provided. In fact, a single ROI that covers the SC blends WM and GM, and it is thus unclear whether was decreased FA caused by a higher proportion of GM with naturally lower FA compared to WM or by actual WM damage. The first 3T NMDC DTI study [28] on $20 \mathrm{HC}$ and 20 NMDC patients without any neurological symptoms and signs (mJOA = 18) excluded also those with radiculopathy, detected lower FA in the entire axial SC at MCL in NMDC patients relative to HC, and corroborated the 1.5T study [25], while it utilized slightly distinct inclusion criteria than the Czech studies $[8,25,26]$. A potential bias from WM and GM combination was further mitigated by additional column-specific analysis that detected decreased FA in the ventral columns of NMDC patients [28]. 
Histopathological reports in SC compression [4,81], which demonstrated damage consistent with malperfusion throughout the territory of compressed anterior spinal artery with restrained blood supplies in the lateral columns, anterior part of dorsal columns and ventral GM horns but not in ventral columns, further emphasize the need for columnand tract-specific studies. Recent 3T tract-specific study detected lower FA and higher MD and RD at MCL in dorsal and lateral tracts in a large cohort of 103 NMDC (mJOA = 18) with or without abnormal electrophysiology and radiculopathy but without myelopathic signs and 21 DCM patients (mJOA < 18) compared to $60 \mathrm{HC}$ with more profound alterations in DCM than NMDC [8]. In agreement with histopathology, GM also showed significant alteration with higher MD, AD, and RD in both NMDC and DCM patients relative to $\mathrm{HC}$ [8]. Lower FA and higher RD at MCL in lateral corticospinal tracts were also found in 16 DCM patients with clinical DCM symptoms without evidence of SC damage on T2-w images compared to 20 controls at 1.5T [82], while no changes were demonstrated in the remaining medial SC part confirming post-mortem studies when delineated demyelination in dorsal and lateral WM tracts $[8,82]$. It is important to note that a stronger magnetic field provides a higher signal-to-noise ratio, which can be translated to higher spatial resolution and shorter acquisition time, while also induces larger susceptibility artifacts [18]. Additionally, the small size of WM tracts and CSF contamination must be compensated using methods for correction of partial volume effect (see Chapter 2.2.5).

Besides direct changes at MCL, studies have also focused on remote neurodegeneration above the compression level in both NMDC and DCM patients. DCM studies $[8,39,40,42,43,75]$ consistently detected decreased FA and increased diffusivity measures at C2/C3 level in dorsal and lateral WM tracts compared to HC, whereas only two DCM studies showed changes also in rostral GM $[8,40]$. The recent NMDC study detected similar changes at C3 level, i.e., decreased FA and increased MD and RD in dorsal and lateral tracts and increased diffusivity measures in GM between DCM and NMDC patients [8]. Thus, outcomes congruently demonstrated remote rostral neurodegeneration of long lateral and dorsal WM tracts and trans-synaptic degeneration of GM in symptomatic DCM patients relative to HC. Incipient remote changes in NMDC patients relative to HC were not detectable by DTI [28] and were, so far, observed only using the multicompartment Ball-and-Sticks model, emphasizing the need for further research and utilization of multi-compartment dMRI models [8].

Importantly, to date, only two DTI studies [27,29] examined NMDC patients longitudinally to monitor progression from NMDC to DCM, and both utilized whole axial SC area (i.e., tissue non-specific) ROI. First study [29] monitored DCM development in 112 NMDC patients with or without the presence of axial pain and/or symptoms or signs of upper extremity monoradiculopathy in 3 years median follow-up using a 1.5T scanner and found no predictive power of DTI. Second 3T study [27] followed-up 66 nonoperatively treated patients with cervical SC compression for an average follow-up of 1.4 years from which 48 had some neurological dysfunction (mJOA $<18$ ) and 18 had neck pain without any neurological signs $(\mathrm{mJOA}=18)$. Although, the study [27] reported that 47 out of 66 patients showed stationary FA and MD that correlated with mJOA score, distinct slice thickness and number of diffusion directions were used over time, making longitudinal FA and MD comparison less reliable since DTI metrics might vary across sequences [83]. However, no work has confirmed DTI as a DCM predictor yet, despite the extensive methodological work in recent years that provides an opportunity for reliable tract-based analyses and holds a promise for accurate longitudinal trials.

\subsubsection{High-order diffusion models}

While higher-order diffusion models have been frequently utilized in DCM studies $[8,50,51,55-57,78]$, only a limited number of works also included NMDC patients $[8,30]$. Higher-order diffusion models usually rely on high angular resolution diffusion imaging [84] and sequences utilizing two or more b-values [85] that require optimized acquisition protocols and processing tools (see Chapter 2.3.1). 
Recently, a multi-shell diffusion protocol with reduced field-of-view allowed estimation of more complex diffusion models such as Ball-and-Sticks model [86] in addition to the conventional DTI model [30]. Multi-compartment Ball-and-Sticks model describes diffusion by a single isotropic and several anisotropic compartments and better characterizes diffusion data than the single-compartment DTI model [87]. The first Ball-and-Sticks study in 33 NMDC patients and 13 HC demonstrated its sensitivity to subtle microstructural changes in both WM and GM [30]. Multi-shell dMRI protocol [30] with b-values of 550 and $1000 \mathrm{~s} / \mathrm{mm}^{2}$ was thereafter used in a large cohort of 103 NMDC (mJOA = 18), 21 DCM patients (mJOA < 18) and $60 \mathrm{HC}$ to delineate changes in dorsal and lateral tracts and GM between NMDC and HC at MCL and also rostrally (Figure 3A). Results suggest superior discriminant power of multi-compartment Ball-and-Sticks model over DTI when depicted abnormalities in $\mathrm{f} 1$ metric (i.e., the primary anisotropic volume fraction), which were not detectable by DTI [8].
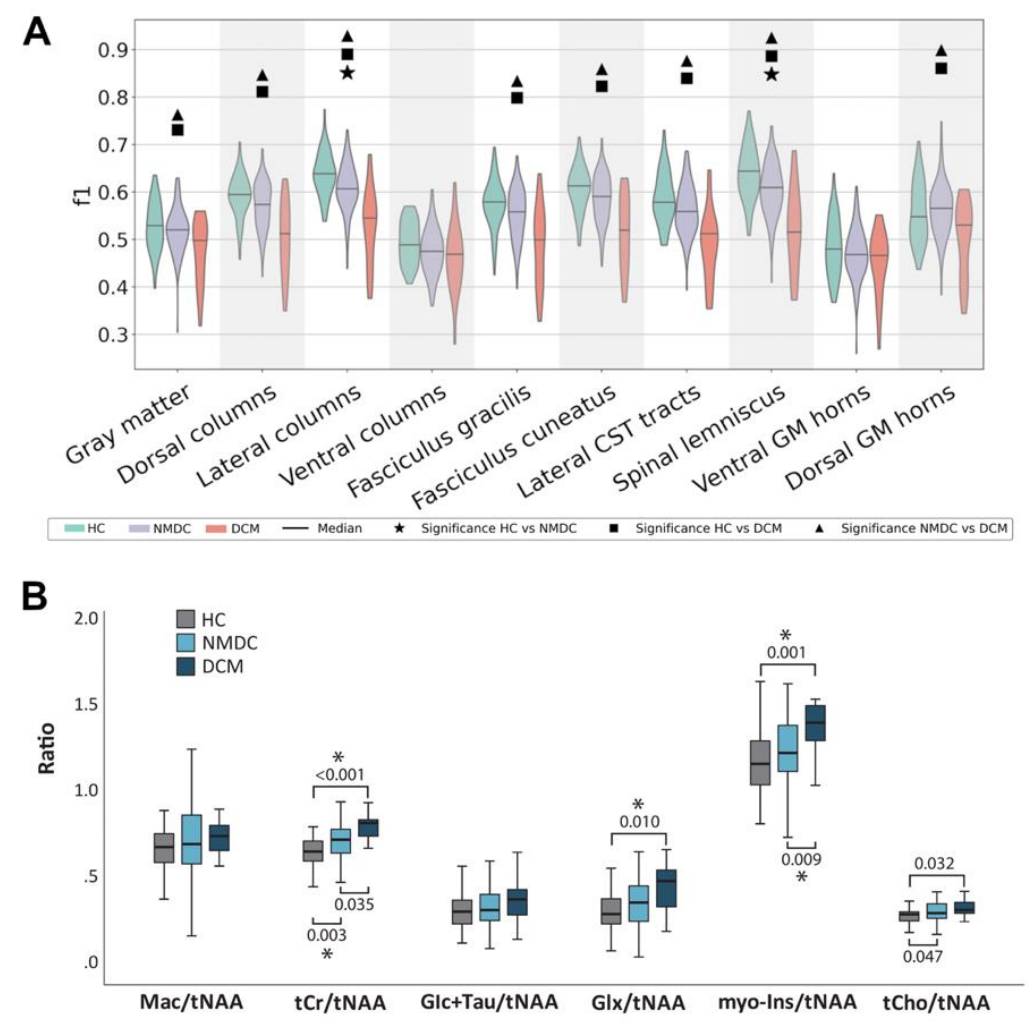

Figure 3. Group differences between NMDC and DCM patients relative to healthy controls (HC). A) Between-group differences in f1 diffusion metric (i.e., primary partial volume fraction of Balland-Sticks model) at C3 above the compression level. Adapted from [8], CC BY-NC (https://creativecommons.org/licenses/by-nc/4.0/); B) Between-group difference in neurometabolies ratios gained from single-voxel magnetic resonance spectroscopy ( $\left.{ }^{1} \mathrm{H}-\mathrm{MRS}\right)$ from above the compression level C2/3. Adapted from [32], CC BY-NC.

Other DCM studies [50,51,55,78] used a three-compartment NODDI model [88], which calculates intracellular volume fraction, isotropic CSF volume fraction, and orientation dispersion index describing the angular variation of axons or dendrites. NODDI was utilized alongside DTI in two retrospective studies $[51,55]$ to monitor surgical outcome in DCM patients and showed increased FA at MCL two weeks after surgery and increased intracellular volume fraction at MCL six months after surgery [51,55]. Authors concluded that findings indicate that neurite density damage in DCM patients might not be irreversible [51]. Other multi-shell techniques such as DKI and QSI provide mean kurtosis, DKI fractional anisotropy (DKI-FA), DKI mean diffusivity (DKI-MD), and root mean 
square displacement metrics [49]. To date, 3T DKI and QSI studies showed lower mean kurtosis along with lower FA and higher root mean square displacement in the entire axial SC ROI in 18 DCM patients with compression relative to $15 \mathrm{DCM}$ patients without the compression [57] and lower mean kurtosis in the GM on affected sides compared to GM on unaffected sides in 13 early DCM patients [56]. Finally, a recent retrospective study ulizing DTI, NODDI and DKI showed lower FA and DKI-FA, and higher DKI-MD, isotropic CSF volume fraction, and orientation dispersion index from the entire axial SC ROI at MCL in $48 \mathrm{DCM}$ patients $(\mathrm{mJOA}<18)$ relative to $36 \mathrm{HC}$ [78]. Isotropic CSF volume fraction, FA, and DKI-FA also correlated with recovery rate calculated based on preoperative and three months follow-up mJOA scales indicating possible usage of these metrics as predictors in surgically treated DCM patients [78].

So far, all published NODDI [51,55,78], DKI [56,57,78], and QSI [57] studies comprised only DCM patients, establishing a further need for the application of innovative dMRI techniques in NMDC patients.

\subsubsection{Intravoxel incoherent motion imaging}

Intravoxel incoherent motion (IVIM) imaging measures microscopic movement of water molecules caused by capillary perfusion using dMRI sequence with low b-values $\left(\leq 300 \mathrm{~mm}^{2} / \mathrm{s}\right)$ to assess flowing blood fraction and pseudo-diffusion coefficient [89]. Pilot IVIM imaging studies in the human SC at 7T in $6 \mathrm{HC}$ [90], and at 3T in 2 DCM patients along with $11 \mathrm{HC}$ [91] depicted higher perfusion in GM compared to WM in HC and impaired perfusion in DCM patients at compression levels. However, interpretation is limited due to the small sample size and possible influence of CSF pulsation [91]. IVIM imaging is a promising technique for future DCM and NMDC studies as post-mortem studies showed that degenerative compression results in hypoperfusion and ischemia in specific WM/GM regions.

\subsubsection{Magnetization transfer}

Magnetization transfer (MT) imaging is based on the exchange of magnetization between protons associated with free water and those linked with immobile macromolecules such as proteins and lipids [18]. The magnetization exchange causes measurable MRI signal attenuation and provides MT ratio (MTR) and MT saturation markers [18,92]. Due to the fact that myelin mainly consists of lipids and proteins, MTR and MT saturation can indirectly measure myelination and are highly sensitive to myelin loss [18,93]. MT imaging was successfully applied in the SC in demyelinating diseases, such as multiple sclerosis [16] or adrenomyeloneuropathy [94] and also in DCM [38,50,95] and NMDC [28] patients.

Martin et al. [28] indeed reported decreased MTR extracted from the entire axial SC ROI in 20 NMDC subjects (mJOA = 18) compared to $20 \mathrm{HC}$ above the compression $(\mathrm{C} 1-\mathrm{C} 3)$ but not at MCL. Column-specific MTR analysis corroborated DTI when demonstrated decreased MTR in ventral columns of NMDC subjects relative to HC [28]. The same group also reported MTR together with FA, cross-sectional area, and T2* WM/GM ratio as useful measures within a composite score for monitoring $26 \mathrm{DCM}$ patients $(\mathrm{mJOA}<18)$ in 13.5 (mean) month follow-up and identified worsening in 11 DCM patients [38]. Another work then showed the predictive value of a combination of the preoperative MTR and shape SC analysis for surgery response and recovery in DCM patients [95]. Finally, a combination of MT imaging and dMRI was used to calculate myelin water fraction, and axon volume fraction in $24 \mathrm{DCM}$ patients compared to $5 \mathrm{HC}$ and reported changes in axon volume fraction between groups in fasciculus gracilis, fasciculus cuneatus, and lateral corticospinal tract [50].

\subsubsection{Magnetic resonance spectroscopy}

Methods of proton magnetic resonance spectroscopy $\left({ }^{1} \mathrm{H}-\mathrm{MRS}\right)$ quantify the neurochemical profile within the spectroscopic volume of interest (i.e., spectroscopic voxel) 
(Figure 1C). Thus, ${ }^{1} \mathrm{H}-\mathrm{MRS}$ provides unique information about neurochemical composition of the neural tissue otherwise inaccessible with conventional imaging methods [96]. The alteration of metabolite profile reflects microstructural or metabolic pathophysiological processes [97]. Metabolite ratios (e.g., myo-inositol (myo-Ins)/N-acetylaspartate (NAA)) could be more sensitive to SC pathology than the metabolites referenced to the water signal particularly when the two metabolites' changes in different direction, for instance, when increased myo-Ins due to the gliosis/astrocytosis compensates the neuronal loss, which per se causes NAA decrease [32,98]. ${ }^{1} \mathrm{H}-\mathrm{MRS}$ in the SC is challenged by the small transversal SC diameters, which is further diminished at the compression level. Therefore, ${ }^{1} \mathrm{H}$-MRS studies in patients with degenerative compression assessed the neurochemical profile only above the stenosis level and observed neurochemical changes rostrally to the compression, likely due to the Wallerian degeneration in patients with clinically manifest myelopathy (i.e., DCM patients). Increased levels of total creatine (tCr)/total NAA (tNAA) [99-102] and total choline (tCho)/tNAA [100,101,103] have been reported in DCM. However, these studies included no more than 35 participants [96,101,104], and did not involve NMDC patients. Recent ${ }^{1} \mathrm{H}-\mathrm{MRS}$ study in 60 NMDC patients with or without electrophysiological changes and radiculopathy but without myelopathic symptoms $(\mathrm{mJOA}$ scale $=18$ ) showed, for the first time, increased $\mathrm{tCr} / \mathrm{tNAA}$ and myo-Ins/tNAA ratios above the stenosis level in NMDC relative to HC pointing to neurochemical changes detectable in clinically silent subjects (Figure 3B) [32]. The high sensitivity of this study arises from superior accuracy in semi-LASER voxel localization [105], improved signal-to-noise ratio at high-field $3 \mathrm{~T}$ scanner, and cardiac triggering minimizing bias from surrounding tissue and cardiac pulsations [32].

Despite the degeneration of afferent tracts, which propagated the changes in DCM patients up to the sensorimotor regions in the brain $[10,11]$, the SC might display earlier alteration of the neurochemical profile and can be a more appropriate target to detect early markers in non-myelopathic compression. Several studies indeed suggested potential predictive value of neurochemical markers when showed correlation between the severity of myelopathy symptoms (mJOA) and metabolite ratios [32,100,106]

The ${ }^{1} \mathrm{H}-\mathrm{MRS}$ sensitivity will benefit from ultra-high fields $[107,108]$, implementation of advanced shimming approaches minimizing anatomically determined pronounced $\mathrm{B}_{0}$-inhomogeinity in the spinal canal [109], and prospective motion correction methods alleviating motion artifacts pronounced during longer acquisitions [110]. In addition, automatization of ${ }^{1} \mathrm{H}-\mathrm{MRS}$ data acquisition, including automatic voxel placement will allow shortening the scan and obtaining operator independent data with the methodology previously implemented for the brain [111]. The methodological improvements along with novel ${ }^{1} \mathrm{H}$-MRS approaches indeed promise to deliver robust neurochemical markers in technically challenging SC region.

\subsection{4. $\mathrm{T} 1$ and $\mathrm{T} 2$ relaxometry}

To date, T1 relaxometry, sensitive to myelination [17], provided contradictory outcomes when it detected lower T1 times in $31 \mathrm{DCM}$ patients on $1.5 \mathrm{~T}$ at compression levels compared to non-stenotic levels above and below [112], but higher T1 times on 3T at compression levels in 22 DCM patients compared to $10 \mathrm{HC}$ [113]. These opposite trends must be further explored with a need for harmonization of field strengths, imaging protocols, and inclusion criteria.

Thus far, myelin water imaging based on T2 relaxometry demonstrated myelin content reduction in dorsal columns in 3T study in 14 DCM patients with pathological somatosensory evoked potentials [114]. Recently, the multicomponent driven equilibrium steady-state observation of T1 and T2 approach utilizing three sequences for estimation of both T1 and T2 times and myelin water fraction was applied in $28 \mathrm{HC}$ to provide myelin imaging atlas and framework for future studies [115].

\subsubsection{Functional MRI}


Functional MRI (fMRI) measures oscillations in neuronal activity utilizing either $\mathrm{T} 2 *$-W sequence sensitive to local magnetic field inhomogeneities related to blood oxygenation level-dependent effect or arterial spin labelling sequences based on arterial water as an endogenous tracer to measure cerebral blood flow [116]. Brain fMRI studies indeed revealed remote changes in activations of motor areas during finger-tapping tasks between DCM patients and HC $[11,117]$, alterations of sensorimotor network in resting-state fMRI in DCM patients [118], the relationship between severity of compression in DCM patients and activation volume in the motor cortex [119], and differences in brain activations in DCM patients with abnormal motor evoked potentials [120] suggesting that SC compression causes secondary brain changes. Spinal cord resting-state fMRI then showed neuronal activity changes in GM horns in 18 DCM patients relative to $25 \mathrm{HC}$ and association of severity of myelopathy with neuronal activity response [121], however, no study has been performed in NMDC [122] yet further emphasizing the need to add fMRI in multimodal SC protocols. Generally, SC fMRI is challenging due to anatomy-related image distortions, low signal-to-noise ratio and physiological movement artifacts [123], which so far limited its use in patients with SC compression.

\subsubsection{Perfusion weighted imaging}

Chronic SC compression in histological and animal studies reduces blood flow in SC arteries and results in malperfusion and SC ischemia [4,81], which are considered vital factors in DCM pathogenesis; however, in-vivo assessments of perfusion deficiency are so far limited $[4,124]$. While perfusion imaging methods, including dynamic susceptibility contrast (DSC) imaging and dynamic contrast-enhanced (DCE) imaging, both utilizing Gd-based contrast agent and arterial spin labelling perfusion imaging, are commonly used in brain studies, there have been sparse SC applications. A recent 3T study in 22 patients with cervical spondylosis with or without myelopathy identified a relationship between DSC markers and anteroposterior diameter and mJOA scale and suggested that the degree of ischemia and hypoxia correlates with compression severity and clinical status, respectively [124]. Another 1.5T DSC study in 14 DCM patients then showed improvement in the SC perfusion after surgical decompression [125] and pseudo-continuous arterial spin labelling, which, unlike DSC and DCE, does not require intravenous contrast agent, revealed secondary alteration of cerebral blood flow perfusion of DCM patients caused by SC compression [126]. 
Table 2. List of studies in patients with non-myelopathic/asymptomatic spinal cord compression utilizing qMRI techniques.

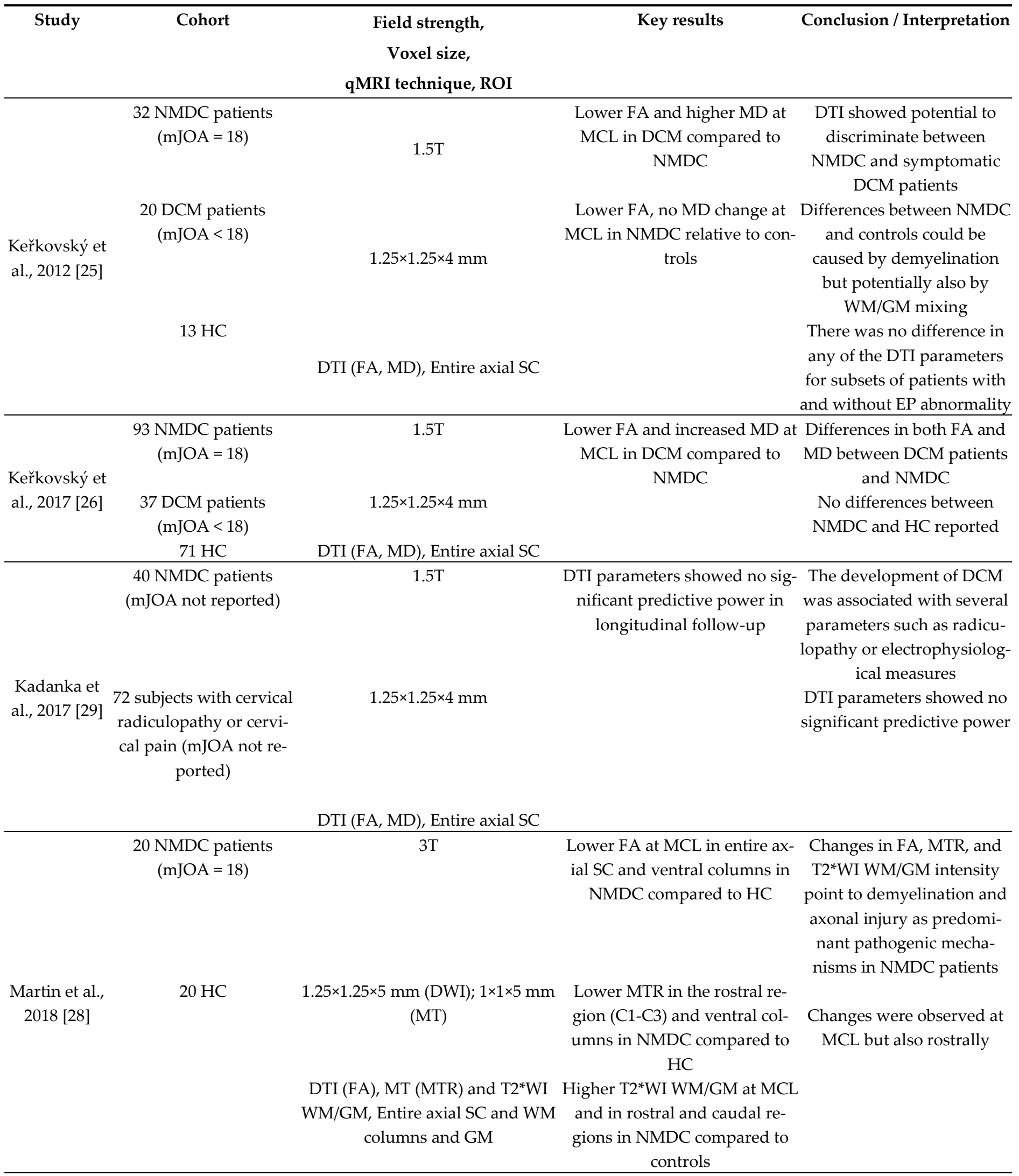




$$
\begin{gathered}
18 \text { NMDC patients } \\
(\mathrm{mJOA}=18)
\end{gathered}
$$

Ellingson et al., 2018 [27] 48 patients with clinical symptoms $(\mathrm{mJOA}<18)$

33 NMDC patients (divided into two groups mild and severe compression)

$13 \mathrm{HC}$

Labounek et al., 2020 [30]

DTI (FA, MD), Ball-and-Sticks model (f1, d), WM-GM difference, and "heuristic" parameters derived from these metrics, WM and GM

103 NMDC patients
$(\mathrm{mJOA}=18)$

21 DCM patients $(\mathrm{mJOA}<18)$

Valošek et al., 2021 [8]
$60 \mathrm{HC}$
$0.65 \times 0.65 \times 3.00 \mathrm{~mm}$ (interpolated)

Changes were detected pre-
dominantly in the GM, dorsal tracts, and lateral tracts at $\mathrm{MCL}$ and rostrally at $\mathrm{C} 3$ level

DTI (FA, MD, AD, RD) and Ball- DCM patients showed changes and-Sticks models (f1, d), WM columns and tracts and GM regions also in the ventral columns compared to $\mathrm{HC}$

dMRI changes correlated with the mJOA scale and reflected electrophysiological findings
Difference in several "heuristic" parameters derived from FA, MD, f1, and d between groups, see the study [30] for details

DTI and Ball-and-Sticks models demonstrated differences between healthy controls and NMDC patients in both WM and GM

Higher MD and $d$ in GM in sion relative to $\mathrm{HC}$

Lower WM-GM difference for MD and $d$ in NMDC with mild and severe compression compared to $\mathrm{HC}$

Lower FA and $\mathrm{f} 1$ and higher $\mathrm{MD}, \mathrm{AD}, \mathrm{RD}$, and $\mathrm{d}$ in NMDC and DCM compared to HC, with more severe changes in DCM compared to NMDC matter, pointing to demye- lination and trans-synaptic degeneration

Above the compression, changes suggest Wallerian degeneration

Changes were more profound in DCM compared to NMDC and HC suggesting progressive changes in patients with compression over time 


\section{NMDC patients}

$(\mathrm{mJOA}=18)$

Horak et al.,

2021 [32]

13 DCM patients

$(\mathrm{mJOA}<18)$

$47 \mathrm{HC}$

${ }^{1} \mathrm{H}-\mathrm{MRS}$
Increased total creatin/tNAA
ratio in NMDC and DCM
${ }^{1} \mathrm{H}-\mathrm{MRS}$ revealed neurochemical changes at the above the compression level $\mathrm{C} 2 / 3$ in both DCM and NMDC compared to HC Neurochemical changes suggest demyelination and Wallerian degeneration DCM compared to $\mathrm{HC}$ myo-Inositol /tNAA ratio in DCM patients correlated with mJOA scale

$102 \mathrm{NMDC}(\mathrm{mJOA}=18) \quad 1.5 \mathrm{~T}$ and $3 \mathrm{~T}$

Logistic model combining compression ratio, cross-sectional Semi-automatic SC detecarea, solidity and torsion de- tion showed lower variabiltected compression with AUC $=$ ity than manual raters 0.947

Automatic compression ratio and cross-sectional area estimation outperforms manual raters
Horakova et

$16 \mathrm{DCM}(\mathrm{mJOA}<18) \quad$ Morphometric metrics (cross-sec-

al., 2022 [33] tional area, compression ratio, solidity, torsion, orientation)

$66 \mathrm{HC}$

AUC, area under the curve; FA, fractional anisotropy; MD, mean diffusivity; AD, axial diffusivity; RD, radial diffusivity; f1, primary partial volume fraction (anisotropic compartment of Ball-and-Sticks model); d, Ball-and-Sticks model diffusivity; MTR, magnetic transfer ratio; ${ }^{1} \mathrm{H}-\mathrm{MRS}$, single-voxel magnetic resonance spectroscopy; WM, white matter; GM, gray matter; mJOA, modified Japanese Orthopaedic Association scale; tNAA, total $\mathrm{N}$-acetylaspartate.

\subsection{Spinal cord MRI data acquisition and processing}

The SC is a small structure with anteroposterior and transverse diameters at $\mathrm{C} 2$ level of 8.8 and $12.4 \mathrm{~mm}$, respectively [127], placed in a bony spinal canal surrounded by CSF with variability in the magnetic susceptibilities that requires optimized acquisition protocols and dedicated analysis tools for accurate and reliable processing [18]. This need is further highlighted in patients with compression with altered SC anatomy.

\subsubsection{Data acquisition}

Suitable sequences with sufficient in-plane resolution, signal-to-noise ratio, and clinically acceptable acquisition times of complete examination under 30-40 min are crucial for tissue-specific analysis. Generally, anisotropic resolution on the order of $1 \times 1 \times 5 \mathrm{~mm}$ is recommended for $\mathrm{dMRI}$ and MT sequences since the $\mathrm{SC}$ is a relatively homogenous structure in the superior-inferior direction and higher slice thickness allows to increase the signal-to-noise ratio and in-plane resolution $[18,83]$. Recently, the SC community released prospectively harmonized spine-generic acquisition protocol [83], allowing multi-center studies [128]. The protocol, which consist of T1-w, T2-w, T2*-w, dMRI, and MT sequences, is freely available for 3T or, with slight modifications, also for 1.5T scanners [83]. Although higher field strength provides higher spatial resolution and a better signal-to-noise ratio, it introduces larger susceptibility artifacts and geometrical distortions, especially for dMRI sequences. Generally, dMRI sequences with reduced field-of-view are recommended over sequences with outer volume suppression to mitigate these artifacts $[30,83,129]$. Cardiac triggering can be then considered to reduce pulsatile CSF flow and to limit motion artifacts and partial volume effect in dMRI [83][46] and also ${ }^{1} \mathrm{H}-\mathrm{MRS}$ [32]. Acquisition of two dMRI sequences with opposite phase-coding and usage of dedicated post-processing tools for correction of motion artifacts and geometrical distortions $[130,131]$ are used across SC studies, despite the fact that these tools are primarily 
designed for the brain and their usage for the SC is the subject of ongoing debate. ${ }^{3}$ An increased signal-to-noise ratio of the 3T dMRI sequences also allows acquiring multi-shell diffusion data with higher b-values, which is crucial for fitting of multi-compartment diffusion models such as NODDI, Ball-and-Sticks, or DKI [83]. Usually, high angular resolution diffusion imaging [84] sequences utilizing diffusion gradient sampling on several whole q-space spheres (i.e., multi-shell diffusion protocols) [85] are employed allowing reliable estimation of the higher-order multi-compartment models.

\subsubsection{Spinal cord data processing}

Robust and reliable automatic data processing saves time, reduces inter-rater variability, and allows reproducible studies. Analyses of the entire axial SC ROI in older NMDC works [25-27,29], which lack spatial resolution and did not allow tracing the spatial origin of the observed microstructural changes, were overcome thanks to probabilistic PAM50 atlas $[47,132]$ and methods for minimizing of partial volume effect $[47,133]$. Thus, more selective analysis and quantification of qMRI markers from individual WM columns, tracts and GM regions can be performed. Tract-specific analysis was successfully used in several recent studies and revealed tissue-specific changes in both DCM and NMDC patients as well as in patients with traumatic spinal cord injury $[8,28,75]$. Alternative approaches for tract delineation are tractography [73,74,134], manually drawn ROIs $[71,72,76]$, or usage of tract-based spatial statistics (TBSS) approach [135]. However, tractography can suffer from inaccuracies caused by severe compression and manually defined ROIs are prone to user bias and take time to draw; thus atlas-based approach is currently preferred $[17,18]$.

The advent of dedicated tools for SC processing implemented in the Spinal Cord Toolbox (SCT) [133] now permits robust automatic segmentations of SC and GM based on convolution neural networks [136,137], processing of structural, dMRI, fMRI and MT images as well as utilizing of probabilistic SC template and PAM50 atlas of WM and GM $[47,132]$, that are compatible with MNI space and can be thus used together with brain templates. Alternative packages such as FMRIB Software Library (FSL) [138], Statistical Parametric Mapping (SPM) software package [139] or JIM (http://www.xinapse.com) designed for brain analysis or dedicated libraries like Dipy [140] or LCmodel [141] for dMRI and MRS analysis, respectively, can also be used for SC data processing. Usually, a combination of tools is used to facilitate multimodal qMRI analysis, for example, SCT is utilized for automatic SC and GM segmentations, morphometric metrics extraction and registration of PAM50 atlas, and is supplemented by FSL or Dipy, which provide tools for fMRI analysis and estimation of higher-order diffusion models. Note that anatomy altered by compression can negatively influence image acquisition and data processing and it is thus necessary to perform quality checks, potential manual correction of segmentations and adjustment of processing parameters (e.g., type of registration). Typical dMRI workflow is summarized in Figure 4.

${ }^{3}$ https://forum.spinalcordmri.org/t/how-to-correct-for-distortions-in-spinal-cord-diffusion-mri-data/326 


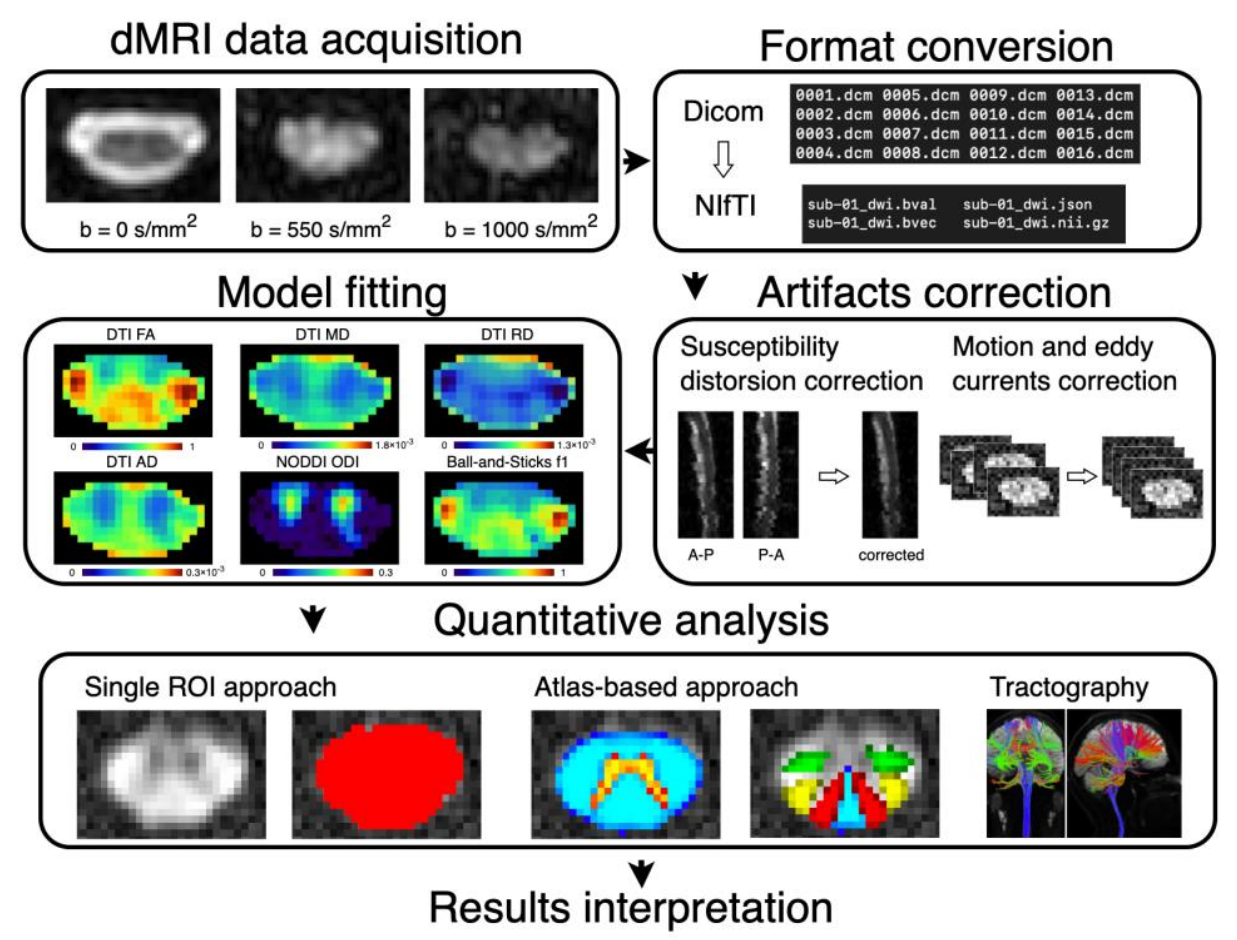

Figure 4. Typical dMRI workflow. dMRI data acquisition is followed by format conversion, usually from DICOM format provided by scanner to NIfTI format [142] supported by many of neuroimaging tools. Subsequent processing pipeline typically includes correction of susceptibility-induced geometrical distortions and motion and eddy currents artifacts and estimation of diffusion model(s). Final quantitative analysis can be done in various ways using single region-of-interest (ROI) approach, atlas-based approach, or tractography. DTI, diffusion tensor imaging; FA, fractional anisotropy; $\mathrm{MD}$, mean diffusivity; $\mathrm{RD}$; radial diffusivity, $\mathrm{AD}$, axial diffusivity; NODDI, neurite orientation dispersion and density imaging; ODI, orientation dispersion index; f1, primary partial volume fraction (anisotropic compartment of Ball-and-Sticks model). Illustration of tractography is reprinted with permission from ref. [18]. Copyright 2014 Elsevier.

\subsection{Quantitative MRI in the SC compression and correlations with clinical outcomes}

A proper estimation of the relationship between qMRI markers and clinical outcomes measured by clinical scores such as mJOA [143] or ASIA [144] scales or electrophysiological measurements is needed to gain insight into clinical relevance of qMRI markers prior to large-scale multicentric longitudinal trials. Whereas studies in DCM patients consistently reported correlations between clinical status assessed by mJOA or ASIA scales and markers derived from dMRI $[8,27,72]$ and MRS [32,100,103], usage of these scales in NMDC patients is limited since these patients are usually asymptomatic and thus without clinical deficits. T2-w signal intensity changes and electrophysiological abnormalities together with signs of radiculopathy were reported as predictors of progression from NMDC into DCM [6], however, the following studies did not find any association with DTI extracted from the entire axial SC ROI [25,29]. While Kadanka et al. [25] indeed did not detect any significant difference in DTI markers from the entire axial SC in NMDC patients with and without electrophysiological abnormality, recent tissue-specific reports demonstrated a relationship between altered electrophysiology and DTI and Ball-andSticks metrics in both NMDC and DCM patients $[8,70]$. Diffusion metrics in lateral motor and dorsal sensory tracts corresponded to alterations in motor and somatosensory evoked potentials, and electromyography corresponded to diffusion metrics in GM $[8,70]$. Finally, Liu et al. [114] found a correlation between the decrease of myelin content in dorsal columns assessed by myelin water imaging and functional deficits (i.e., prolonged cortical somatosensory evoked potential latencies) in DCM patients. Recently, contact heat evoked potentials demonstrated high sensitivity in DCM patients [145] and might be promising in future longitudinal studies besides qMRI markers. 


\section{Conclusion and future directions}

While previous studies clearly confirmed alterations in SC qMRI in both NMDC and DCM patients relative to $\mathrm{HC}$, the results showed some inconsistencies due to distinctions in scanners' field strength, acquisition protocols, and data post-processing. Also, unification of the inclusion criteria is particularly needed for NMDC individuals as some studies include only those without radiculopathy [28] while others also incorporated NMDC subjects with radiculopathy $[8,25,26]$.

To date, DTI studies performed at both 1.5T and 3T consistently detected lower FA and higher MD at MCL in NMDC and DCM patients relative to $\mathrm{HC}$ with more progressive changes in DCM compared to NMDC; these changes are likely caused by edema, deficits in the degree of myelination, axonal packing and axon size, or co-linearity of fiber organization. Some also found deficits in RD and MTR pointing to the demyelination $[8,28,82]$ and $\mathrm{AD}$ alteration due to axonal injury as the primary alteration at MCL [8]. Rostral secondary changes in DCM patients presented as lower FA and higher diffusivity measures in dorsal columns and lateral corticospinal tracts and changes in ${ }^{1} \mathrm{H}-\mathrm{MRS}$ ratios at $\mathrm{C} 2 / 3$ level point to remote Wallerian degeneration above the compression level $[8,32,39,40,42,43,75,103,104]$. Subtle remote changes at C2/3 level between NMDC and HC were then revealed by the multi-compartment Ball-and-Sticks diffusion model, ${ }^{1} \mathrm{H}-\mathrm{MRS}$, and MTR $[8,28,32]$. Moreover, brain fMRI and ${ }^{1} \mathrm{H}-\mathrm{MRS}$ studies in DCM patients showed secondary changes even in the brain, suggesting alterations in neuronal activations and brain plasticity caused by chronic SC compression [10,11]. Existing studies also showed the relationship between clinical impairments assessed by clinical scales and microstructural degeneration measured using qMRI $[8,27,32,72,100,103]$. Finally, several works provided evidence of the relationship between functional impairments measured using electrophysiology and qMRI derived metrics [8,70,114].

The widespread availability of $3 \mathrm{~T}$ scanners in the clinical practice also further emphasizes the need to harmonize protocols across scanners and vendors to estimate normative values, which was so far limited by the usage of different sequences and acquisition parameters. Indeed, the release of the spine-generic acquisition protocol [83] provided a critical step forward for the upcoming longitudinal multicentric studies with the promise of normative quantitative values. $3 \mathrm{~T}$ protocols, which minimize susceptible artifacts (i.e., reduced field-of-view technique), while allowing to benefit from increased signal-to-noise ratio compared to lower fields, are particularly important for methods such as $\mathrm{dMRI}$ and ${ }^{1} \mathrm{H}$-MRS [18]. High in-plane resolution $\leq 1 \times 1 \times 5 \mathrm{~mm}$ of recent dMRI and MT sequences $[8,28,43,46]$ allowed tissue- and tract-specific analysis, which must be accompanied by partial volume correction to rule out partial volume effect, though. Lastly, pilot studies at 7T showed promising results for future research that might further increase our understanding of metabolic and microstructural damage, and the utilization will require further sequence development and usage of dedicated coils.

In conclusion, whereas high-resolution 3T qMRI with tissue- and tract-specific analysis supplemented by electrophysiological measures and clinical scales indeed showed ongoing alteration of SC microstructure even in NMDC patients, longitudinal and multicentric studies with optimized protocols are critical for future NMDC research to monitor possible progression to clinically manifest DCM. The application of qMRI as possible predictors of progression from NMDC to DCM must be further verified by an estimation of normative values for the clinical practice; however, such goal requires harmonization of the SC protocols across scanners and vendors.

Author Contributions: Conceptualization, J.V., P.B., J.B. and A.S.; writing - original draft preparation, J.V. and A.S.; writing - review and editing, J.V., P.B., M.K., P.H., J.B. and A.S.; visualization, J.V., P.B. and A.S.; supervision, P.B., M.K., P.H., J.B. and A.S.; funding acquisition, P.H. and J.B. All authors have read and agreed to the published version of the manuscript.

Funding: The core facility Multimodal and Functional Imaging Laboratory, Masaryk University, CEITEC, supported by the MEYS CR (LM2018129 Czech-Biolmaging), is acknowledged. This 
research is funded by the Czech Health Research Council grants NV18-04-00159 and NU22-0400024, and by the Ministry of Health of the Czech Republic project for conceptual development in research organizations, ref. 65269705 (University Hospital, Brno, Czech Republic) and ref. 00098892 (University Hospital Olomouc, Czech Republic). JV has received “Aktion Österreich-Tschechien, AÖCZ-Semesterstipendien" scholarship MPC-2020-00013 from the Austrian Agency for International Cooperation in Education and Research (OeAD-GmbH), Mobility Programmes, Bilateral and Multilateral Cooperation (MPC) financed by Federal Ministry of Education, Science and Research (BMBWF) of Austria. AS has received funding from the European Union's Horizon 2020 research and innovation programme under the Marie Skłodowska-Curie grant agreement no. 794986.

Acknowledgments: We thank associate professor Eva Vlčková from University Hospital Brno for her rigorous revisions of the text and providing valuable advice.

Conflicts of Interest: The authors declare no conflict of interest.

\section{References}

1. Smith, S.S.; Stewart, M.E.; Davies, B.M.; Kotter, M.R.N. The Prevalence of Asymptomatic and Symptomatic Spinal Cord Compression on Magnetic Resonance Imaging: A Systematic Review and Meta-analysis. Glob. Spine J. 2021, 11, 597-607, doi:10.1177/2192568220934496.

2. Kovalova, I.; Kerkovsky, M.; Kadanka, Z.; Kadanka, Z.; Nemec, M.; Jurova, B.; Dusek, L.; Jarkovsky, J.; Bednarik, J. Prevalence and imaging characteristics of nonmyelopathic and myelopathic spondylotic cervical cord compression. Spine (Phila. Pa. 1976). 2016, 41, 1908-1916, doi:10.1097/BRS.0000000000001842.

3. Adamova, B.; Bednarik, J.; Andrasinova, T.; Kovalova, I.; Kopacik, R.; Jabornik, M.; Kerkovsky, M.; Jakubcova, B.; Jarkovsky, J. Does lumbar spinal stenosis increase the risk of spondylotic cervical spinal cord compression? Eur. Spine J. 2015, 24, 29462953, doi:10.1007/s00586-015-4049-0.

4. Badhiwala, J.H.; Ahuja, C.S.; Akbar, M.A.; Witiw, C.D.; Nassiri, F.; Furlan, J.C.; Curt, A.; Wilson, J.R.; Fehlings, M.G. Degenerative cervical myelopathy - update and future directions. Nat. Rev. Neurol. 2020, 16, 108-124, doi:10.1038/s41582019-0303-0.

5. Bednarik, J.; Kadanka, Z.; Dusek, L.; Novotny, O.; Surelova, D.; Urbanek, I.; Prokes, B. Presymptomatic spondylotic cervical cord compression. Spine (Phila. Pa. 1976). 2004, 29, 2260-2269, doi:10.1097/01.brs.0000142434.02579.84.

6. Bednarik, J.; Kadanka, Z.; Dusek, L.; Kerkovsky, M.; Vohanka, S.; Novotny, O.; Urbanek, I.; Kratochvilova, D. Presymptomatic spondylotic cervical myelopathy: An updated predictive model. Eur. Spine J. 2008, 17, 421-431, doi:10.1007/s00586-008-0585-1.

7. Fehlings, M.G.; Tetreault, L.A.; Riew, K.D.; Middleton, J.W.; Aarabi, B.; Arnold, P.M.; Brodke, D.S.; Burns, A.S.; Carette, S.; Chen, R.; et al. A Clinical Practice Guideline for the Management of Patients With Degenerative Cervical Myelopathy: Recommendations for Patients With Mild, Moderate, and Severe Disease and Nonmyelopathic Patients With Evidence of Cord Compression. Glob. Spine J. 2017, 7, 70S-83S, doi:10.1177/2192568217701914.

8. Valošek, J.; Labounek, R.; Horák, T.; Horáková, M.; Bednař́́k, P.; Keřkovský, M.; Kočica, J.; Rohan, T.; Lenglet, C.; CohenAdad, J.; et al. Diffusion magnetic resonance imaging reveals tract-specific microstructural correlates of electrophysiological impairments in non-myelopathic and myelopathic spinal cord compression. Eur. J. Neurol. 2021, 28, 3784-3797, doi:10.1111/ene.15027.

9. David, G.; Mohammadi, S.; Martin, A.R.; Cohen-Adad, J.; Weiskopf, N.; Thompson, A.; Freund, P. Traumatic and nontraumatic spinal cord injury: pathological insights from neuroimaging. Nat. Rev. Neurol. 2019, 15, 718-731, doi:10.1038/s41582-019-0270-5.

10. Kowalczyk, I.; Duggal, N.; Bartha, R. Proton magnetic resonance spectroscopy of the motor cortex in cervical myelopathy. Brain 2012, 135, 461-468, doi:10.1093/brain/awr328.

11. Bernabéu-Sanz, Á.; Mollá-Torró, J.V.; López-Celada, S.; Moreno López, P.; Fernández-Jover, E. MRI evidence of brain atrophy, 
white matter damage, and functional adaptive changes in patients with cervical spondylosis and prolonged spinal cord compression. Eur. Radiol. 2020, 30, 357-369, doi:10.1007/s00330-019-06352-z.

12. Witiw, C.D.; Mathieu, F.; Nouri, A.; Fehlings, M.G. Clinico-Radiographic Discordance: An Evidence-Based Commentary on the Management of Degenerative Cervical Spinal Cord Compression in the Absence of Symptoms or With Only Mild Symptoms of Myelopathy. Glob. Spine J. 2018, 8, 527-534, doi:10.1177/2192568217745519.

13. Wilson, J.R.; Barry, S.; Fischer, D.J.; Skelly, A.C.; Arnold, P.M.; Riew, K.D.; Shaffrey, C.I.; Traynelis, V.C.; Fehlings, M.G. Frequency, Timing, and Predictors of Neurological Dysfunction in the Nonmyelopathic Patient With Cervical Spinal Cord Compression, Canal Stenosis, and/or Ossification of the Posterior Longitudinal Ligament. Spine (Phila. Pa. 1976). 2013, 38, S37-S54, doi:10.1097/BRS.0b013e3182a7f2e7.

14. WFNS Cervical Spondylotic Myelopathy Available online: http://wfns-spine.org/recom-cervical-spondylotic-myelopathy-1.

15. Oh, T.; Lafage, R.; Lafage, V.; Protopsaltis, T.; Challier, V.; Shaffrey, C.; Kim, H.J.; Arnold, P.; Chapman, J.; Schwab, F.; et al. Comparing Quality of Life in Cervical Spondylotic Myelopathy with Other Chronic Debilitating Diseases Using the Short Form Survey 36-Health Survey. World Neurosurg. 2017, 106, 699-706, doi:10.1016/j.wneu.2016.12.124.

16. Martin, A.R.; Aleksanderek, I.; Cohen-Adad, J.; Tarmohamed, Z.; Tetreault, L.; Smith, N.; Cadotte, D.W.; Crawley, A.; Ginsberg, H.; Mikulis, D.J.; et al. Translating state-of-the-art spinal cord MRI techniques to clinical use: A systematic review of clinical studies utilizing DTI, MT, MWF, MRS, and fMRI. NeuroImage Clin. 2016, 10, 192-238, doi:10.1016/j.nicl.2015.11.019.

17. Cohen-Adad, J. Microstructural imaging in the spinal cord and validation strategies. Neuroimage 2018, 182, 169-183, doi:10.1016/j.neuroimage.2018.04.009.

18. Cohen-Adad, J.; Wheeler-Kingshott, C. Quantitative MRI of the Spinal Cord; Elsevier, 2014; ISBN 9780123969736.

19. Baptiste, D.C.; Fehlings, M.G. Pathophysiology of cervical myelopathy. Spine J. 2006, 6, 190-197, doi:10.1016/j.spinee.2006.04.024.

20. Akter, F.; Yu, X.; Qin, X.; Yao, S.; Nikrouz, P.; Syed, Y.A.; Kotter, M. The Pathophysiology of Degenerative Cervical Myelopathy and the Physiology of Recovery Following Decompression. Front. Neurosci. 2020, 14, doi:10.3389/fnins.2020.00138.

21. Akter, F.; Kotter, M. Pathobiology of Degenerative Cervical Myelopathy. Neurosurg. Clin. N. Am. 2018, 29, 13-19, doi:10.1016/j.nec.2017.09.015.

22. Tu, J.; Vargas Castillo, J.; Das, A.; Diwan, A.D. Degenerative Cervical Myelopathy: Insights into Its Pathobiology and Molecular Mechanisms. J. Clin. Med. 2021, 10, 1214, doi:10.3390/jcm10061214.

23. Guan, X.; Fan, G.; Wu, X.; Gu, G.; Gu, X.; Zhang, H.; He, S. Diffusion Tensor Imaging Studies of Cervical Spondylotic Myelopathy: A Systemic Review and Meta-Analysis. PLoS One 2015, 10, e0117707, doi:10.1371/journal.pone.0117707.

24. Ellingson, B.M.; Salamon, N.; Holly, L.T. Advances in MR imaging for cervical spondylotic myelopathy. Eur. Spine J. 2015, 24, 197-208, doi:10.1007/s00586-013-2915-1.

25. Keřkovský, M.; Bednař́́k, J.; Dušek, L.; Šprláková-Puková, A.; Urbánek, I.; Mechl, M.; Válek, V.; Kadaňka, Z. Magnetic resonance diffusion tensor imaging in patients with cervical spondylotic spinal cord compression: Correlations between clinical and electrophysiological findings. Spine (Phila. Pa. 1976). 2012, 37, 48-56, doi:10.1097/BRS.0b013e31820e6c35.

26. Keřkovský, M.; Bednařík, J.; Jurová, B.; Dušek, L.; Kadaňka, Z.; Kadaňka, Z.; Němec, M.; Koval’ová, I.; Šprláková-Puková, A.; Mechl, M. Spinal Cord MR Diffusion Properties in Patients with Degenerative Cervical Cord Compression. J. Neuroimaging 2017, 27, 149-157, doi:10.1111/jon.12372.

27. Ellingson, B.M.; Salamon, N.; Woodworth, D.C.; Yokota, H.; Holly, L.T. Reproducibility, temporal stability, and functional correlation of diffusion MR measurements within the spinal cord in patients with asymptomatic cervical stenosis or cervical myelopathy. J. Neurosurg. Spine 2018, 28, 472-480, doi:10.3171/2017.7.SPINE176.

28. Martin, A.R.; De Leener, B.; Cohen-Adad, J.; Cadotte, D.W.; Nouri, A.; Wilson, J.R.; Tetreault, L.; Crawley, A.P.; Mikulis, D.J.; Ginsberg, H.; et al. Can microstructural MRI detect subclinical tissue injury in subjects with asymptomatic cervical spinal 
cord compression? A prospective cohort study. BMJ Open 2018, 8, e019809, doi:10.1136/bmjopen-2017-019809.

29. Kadanka, Z.; Adamova, B.; Kerkovsky, M.; Kadanka, Z.; Dusek, L.; Jurova, B.; Vlckova, E.; Bednarik, J. Predictors of symptomatic myelopathy in degenerative cervical spinal cord compression. Brain Behav. 2017, 7, e00797, doi:10.1002/brb3.797.

30. Labounek, R.; Valošek, J.; Horák, T.; Svátková, A.; Bednařík, P.; Vojtíšek, L.; Horáková, M.; Nestrašil, I.; Lenglet, C.; CohenAdad, J.; et al. HARDI-ZOOMit protocol improves specificity to microstructural changes in presymptomatic myelopathy. Sci. Rep. 2020, 10, 17529, doi:10.1038/s41598-020-70297-3.

31. Kadanka, Z.; Kadanka, Z.; Skutil, T.; Vlckova, E.; Bednarik, J. Walk and Run Test in Patients with Degenerative Compression of the Cervical Spinal Cord. J. Clin. Med. 2021, 10, 927, doi:10.3390/jcm10050927.

32. Horak, T.; Horakova, M.; Svatkova, A.; Kadanka, Z.; Kudlicka, P.; Valosek, J.; Rohan, T.; Kerkovsky, M.; Vlckova, E.; Kadanka, Z.; et al. In vivo Molecular Signatures of Cervical Spinal Cord Pathology in Degenerative Compression. J. Neurotrauma 2021, 38, 2999-3010, doi:10.1089/neu.2021.0151.

33. Horáková, M.; Horák, T.; Valošek, J.; Rohan, T.; Koritáková, E.; Dostál, M.; Kocíca, J.; Skutil, T.; Kerkkovský, M.; Kadanka Jr, Z.; et al. Semi-automated detection of cervical spinal cord compression with the Spinal Cord Toolbox. Quant. Imaging Med. Surg. 2022, 12, 2261-2279, doi:10.21037/qims-21-782.

34. Nouri, A.; Martin, A.R.; Kato, S.; Reihani-Kermani, H.; Riehm, L.E.; Fehlings, M.G. The Relationship between MRI Signal Intensity Changes, Clinical Presentation, and Surgical Outcome in Degenerative Cervical Myelopathy. Spine (Phila. Pa. 1976). 2017, 42, 1851-1858, doi:10.1097/BRS.0000000000002234.

35. Martin, A.R.; Tetreault, L.; Nouri, A.; Curt, A.; Freund, P.; Rahimi-Movaghar, V.; Wilson, J.R.; Fehlings, M.G.; Kwon, B.K.; Harrop, J.S.; et al. Imaging and Electrophysiology for Degenerative Cervical Myelopathy [AO Spine RECODE-DCM Research Priority Number 9]. Glob. Spine J. 2022, 12, 130S-146S, doi:10.1177/21925682211057484.

36. Nouri, A.; Martin, A.R.; Mikulis, D.; Fehlings, M.G. Magnetic resonance imaging assessment of degenerative cervical myelopathy: A review of structural changes and measurement techniques. Neurosurg. Focus 2016, 40, E5, doi:10.3171/2016.3.FOCUS1667.

37. Kato, F.; Yukawa, Y.; Suda, K.; Yamagata, M.; Ueta, T. Normal morphology, age-related changes and abnormal findings of the cervical spine. Part II: Magnetic resonance imaging of over 1,200 asymptomatic subjects. Eur. Spine J. 2012, 21, 1499-1507, doi:10.1007/s00586-012-2176-4.

38. Martin, A.R.; De Leener, B.; Cohen-Adad, J.; Kalsi-Ryan, S.; Cadotte, D.W.; Wilson, J.R.; Tetreault, L.; Nouri, A.; Crawley, A.; Mikulis, D.J.; et al. Monitoring for myelopathic progression with multiparametric quantitative MRI. PLoS One 2018, 13, e0195733, doi:10.1371/journal.pone.0195733.

39. Grabher, P.; Mohammadi, S.; David, G.; Freund, P. Neurodegeneration in the Spinal Ventral Horn Prior to Motor Impairment in Cervical Spondylotic Myelopathy. J. Neurotrauma 2017, 34, 2329-2334, doi:10.1089/neu.2017.4980.

40. Grabher, P.; Mohammadi, S.; Trachsler, A.; Friedl, S.; David, G.; Sutter, R.; Weiskopf, N.; Thompson, A.J.; Curt, A.; Freund, P. Voxel-based analysis of grey and white matter degeneration in cervical spondylotic myelopathy. Sci. Rep. 2016, 6, 24636, doi:10.1038/srep24636.

41. Valošek, J.; Bednařík, P.; Horák, T.; Horáková, M.; Svátková, A.; Labounek, R.; Hluštík, P.; Bednařík, J. Cervical Spinal Cord Atrophy Above Level of Asymptomatic Degenerative Cervical Cord Compression. OHBM 2020.

42. Vallotton, K.; David, G.; Hupp, M.; Pfender, N.; Cohen-Adad, J.; Fehlings, M.G.; Samson, R.S.; Wheeler-Kingshott, C.A.M.G.; Curt, A.; Freund, P.; et al. Tracking White and Gray Matter Degeneration along the Spinal Cord Axis in Degenerative Cervical Myelopathy. J. Neurotrauma 2021, 38, 2978-2987, doi:10.1089/neu.2021.0148.

43. David, G.; Vallotton, K.; Hupp, M.; Curt, A.; Freund, P.; Seif, M. Extent of Cord Pathology in the Lumbosacral Enlargement in Non-Traumatic versus Traumatic Spinal Cord Injury. J. Neurotrauma 2022, doi:10.1089/neu.2021.0389.

44. Papinutto, N.; Asteggiano, C.; Bischof, A.; Gundel, T.J.; Caverzasi, E.; Stern, W.A.; Bastianello, S.; Hauser, S.L.; Henry, R.G. Intersubject Variability and Normalization Strategies for Spinal Cord Total Cross-Sectional and Gray Matter Areas. J. 
Neuroimaging 2020, 30, 110-118, doi:10.1111/jon.12666.

45. Bédard, S.; Cohen-Adad, J. Normalizing automatic spinal cord cross-sectional area measures. bioRxiv 2021, doi:10.1101/2021.09.30.462636.

46. Martin, A.R.; De Leener, B.; Cohen-Adad, J.; Cadotte, D.W.; Kalsi-Ryan, S.; Lange, S.F.; Tetreault, L.; Nouri, A.; Crawley, A.; Mikulis, D.J.; et al. Clinically Feasible Microstructural MRI to Quantify Cervical Spinal Cord Tissue Injury Using DTI, MT, and T2*-Weighted Imaging: Assessment of Normative Data and Reliability. AJNR. Am. J. Neuroradiol. 2017, 38, 1257-1265, doi:10.3174/ajnr.A5163.

47. Lévy, S.; Benhamou, M.; Naaman, C.; Rainville, P.; Callot, V.; Cohen-Adad, J. White matter atlas of the human spinal cord with estimation of partial volume effect. Neuroimage 2015, 119, 262-271, doi:10.1016/j.neuroimage.2015.06.040.

48. Johansen-Berg, H.; Behrens, T.E.J. Diffusion MRI: From Quantitative Measurement to In vivo Neuroanatomy: Second Edition; Elsevier Science, 2013; ISBN 9780123964601.

49. Mori, S.; Tournier, J.-D. Introduction to Diffusion Tensor Imaging: And Higher Order Models; 2 edition.; Academic Press: Amsterdam, 2014; ISBN 978-0123983985.

50. Hori, M.; Hagiwara, A.; Fukunaga, I.; Ueda, R.; Kamiya, K.; Suzuki, Y.; Liu, W.; Murata, K.; Takamura, T.; Hamasaki, N.; et al. Application of Quantitative Microstructural MR Imaging with Atlas-based Analysis for the Spinal Cord in Cervical Spondylotic Myelopathy. Sci. Rep. 2018, 8, 5213, doi:10.1038/s41598-018-23527-8.

51. Iwama, T.; Ohba, T.; Okita, G.; Ebata, S.; Ueda, R.; Motosugi, U.; Onishi, H.; Haro, H.; Hori, M. Utility and validity of neurite orientation dispersion and density imaging with diffusion tensor imaging to quantify the severity of cervical spondylotic myelopathy and assess postoperative neurological recovery. Spine J. 2020, 20, 417-425, doi:10.1016/j.spinee.2019.10.019.

Grussu, F.; Schneider, T.; Zhang, H.; Alexander, D.C.; Wheeler-Kingshott, C.A.M. Single-shell diffusion MRI NODDI with in vivo cervical cord data. In Proceedings of the ISMRM; 2014; Vol. 1716.

53. Grussu, F.; Schneider, T.; Zhang, H.; Alexander, D.C.; Wheeler-Kingshott, C.A.M. Neurite orientation dispersion and density imaging of the healthy cervical spinal cord in vivo. Neuroimage 2015, 111, 590-601, doi:10.1016/j.neuroimage.2015.01.045.

54. Grussu, F.; Schneider, T.; Tur, C.; Yates, R.L.; Tachrount, M.; Ianus B, A.; Yiannakas, M.C.; Newcombe, J.; Zhang, H.; Alexander, D.C.; et al. Neurite dispersion: a new marker of multiple sclerosis spinal cord pathology? Ann. Clin. Transl. Neurol. 2017, 4, 663-679, doi:10.1002/acn3.445.

55. Okita, G.; Ohba, T.; Takamura, T.; Ebata, S.; Ueda, R.; Onishi, H.; Haro, H.; Hori, M. Application of neurite orientation dispersion and density imaging or diffusion tensor imaging to quantify the severity of cervical spondylotic myelopathy and to assess postoperative neurologic recovery. Spine J. 2018, 18, 268-275, doi:10.1016/j.spinee.2017.07.007.

56. Hori, M.; Tsutsumi, S.; Yasumoto, Y.; Ito, M.; Suzuki, M.; Tanaka, F.S.; Kyogoku, S.; Nakamura, M.; Tabuchi, T.; Fukunaga, I.; et al. Cervical spondylosis: Evaluation of microstructural changes in spinal cord white matter and gray matter by diffusional kurtosis imaging. Magn. Reson. Imaging 2014, 32, 428-432, doi:10.1016/j.mri.2014.01.018.

57. Hori, M.; Fukunaga, I.; Masutani, Y.; Nakanishi, A.; Shimoji, K.; Kamagata, K.; Asahi, K.; Hamasaki, N.; Suzuki, Y.; Aoki, S. New diffusion metrics for spondylotic myelopathy at an early clinical stage. Eur. Radiol. 2012, 22, 1797-1802, doi:10.1007/s00330-012-2410-9.

58. Duval, T.; McNab, J.A.; Setsompop, K.; Witzel, T.; Schneider, T.; Huang, S.Y.; Keil, B.; Klawiter, E.C.; Wald, L.L.; Cohen-Adad, J. In vivo mapping of human spinal cord microstructure at 300mT/m. Neuroimage 2015, 118, 494-507, doi:10.1016/j.neuroimage.2015.06.038.

59. Duval, T.; Smith, V.; Stikov, N.; Klawiter, E.C.; Cohen-Adad, J. Scan-rescan of axcaliber, macromolecular tissue volume, and g-ratio in the spinal cord. Magn. Reson. Med. 2018, 79, 2759-2765, doi:10.1002/mrm.26945.

60. Duval, T.; Lévy, S.; Stikov, N.; Campbell, J.; Mezer, A.; Witzel, T.; Keil, B.; Smith, V.; Wald, L.L.; Klawiter, E.; et al. g-Ratio weighted imaging of the human spinal cord in vivo. Neuroimage 2017, 145, 11-23, doi:10.1016/j.neuroimage.2016.09.018.

61. Farrell, J.A.D.; Smith, S.A.; Gordon-Lipkin, E.M.; Reich, D.S.; Calabresi, P.A.; Van Zijl, P.C.M. High b-value q-space diffusion- 
weighted MRI of the human cervical spinal cord in vivo: Feasibility and application to multiple sclerosis. Magn. Reson. Med. 2008, 59, 1079-1089, doi:10.1002/mrm.21563.

62. Hori, M.; Motosug, U.; Fatima, Z.; Ishigame, K.; Araki, T. Mean displacement map of spine and spinal cord disorders using high b-value q-space imaging-feasibility study. Acta radiol. 2011, 52, 1155-1158, doi:10.1258/ar.2011.110226.

63. Cohen-Adad, J.; Descoteaux, M.; Rossignol, S.; Hoge, R.D.; Deriche, R.; Benali, H. Detection of multiple pathways in the spinal cord using q-ball imaging. Neuroimage 2008, 42, 739-749, doi:10.1016/j.neuroimage.2008.04.243.

64. Lee, J.W.; Kim, J.H.; Park, J. Bin; Park, K.W.; Yeom, J.S.; Lee, G.Y.; Kang, H.S. Diffusion tensor imaging and fiber tractography in cervical compressive myelopathy: Preliminary results. Skeletal Radiol. 2011, 40, 1543-1551, doi:10.1007/s00256-011-1161-z.

65. Ellingson, B.M.; Salamon, N.; Grinstead, J.W.; Holly, L.T. Diffusion tensor imaging predicts functional impairment in mildto-moderate cervical spondylotic myelopathy. Spine J. 2014, 14, 2589-2597, doi:10.1016/j.spinee.2014.02.027.

66. Wen, C.Y.; Cui, J.L.; Liu, H.S.; Mak, K.C.; Cheung, W.Y.; Luk, K.D.K.; Hu, Y. Is diffusion anisotropy a biomarker for disease severity and surgical prognosis of cervical spondylotic myelopathy. Radiology 2014, 270, 197-204, doi:10.1148/radiol.13121885. Jones, J.G.A.; Cen, S.Y.; Lebel, R.M.; Hsieh, P.C.; Law, M. Diffusion tensor imaging correlates with the clinical assessment of disease severity in cervical spondylotic myelopathy and predicts outcome following surgery. Am. J. Neuroradiol. 2013, 34, 471-478, doi:10.3174/ajnr.A3199.

68. Uda, T.; Takami, T.; Tsuyuguchi, N.; Sakamoto, S.; Yamagata, T.; Ikeda, H.; Nagata, T.; Ohata, K. Assessment of cervical spondylotic myelopathy using diffusion tensor magnetic resonance imaging parameter at 3.0 tesla. Spine (Phila. Pa. 1976). 2013, 38, 407-414, doi:10.1097/BRS.0b013e31826f25a3.

69. Banaszek, A.; Bladowska, J.; Szewczyk, P.; Podgórski, P.; Sąsiadek, M. Usefulness of diffusion tensor MR imaging in the assessment of intramedullary changes of the cervical spinal cord in different stages of degenerative spine disease. Eur. Spine J. 2014, 23, 1523-1530, doi:10.1007/s00586-014-3323-x.

70. Wen, C.Y.; Cui, J.L.; Mak, K.C.; Luk, K.D.K.; Hu, Y. Diffusion tensor imaging of somatosensory tract in cervical spondylotic myelopathy and its link with electrophysiological evaluation. Spine J. 2014, 14, 1493-1500, doi:10.1016/j.spinee.2013.08.052.

71. Maki, S.; Koda, M.; Ota, M.; Oikawa, Y.; Kamiya, K.; Inada, T.; Furuya, T.; Takahashi, K.; Masuda, Y.; Matsumoto, K.; et al. Reduced Field-of-View Diffusion Tensor Imaging of the Spinal Cord Shows Motor Dysfunction of the Lower Extremities in Patients With Cervical Compression Myelopathy. Spine (Phila. Pa. 1976). 2018, 43, 89-96, doi:10.1097/BRS.0000000000001123. Maki, S.; Koda, M.; Saito, J.; Takahashi, S.; Inada, T.; Kamiya, K.; Ota, M.; Iijima, Y.; Masuda, Y.; Matsumoto, K.; et al. TractSpecific Diffusion Tensor Imaging Reveals Laterality of Neurological Symptoms in Patients with Cervical Compression Myelopathy. World Neurosurg. 2016, 96, 184-190, doi:10.1016/j.wneu.2016.08.129.

73. Cui, J.L.; Li, X.; Chan, T.Y.; Mak, K.C.; Luk, K.D.K.; Hu, Y. Quantitative assessment of column-specific degeneration in cervical spondylotic myelopathy based on diffusion tensor tractography. Eur. Spine J. 2015, 24, 41-47, doi:10.1007/s00586-0143522-5.

74. Budzik, J.F.; Balbi, V.; Le Thuc, V.; Duhamel, A.; Assaker, R.; Cotten, A. Diffusion tensor imaging and fibre tracking in cervical spondylotic myelopathy. Eur. Radiol. 2011, 21, 426-433, doi:10.1007/s00330-010-1927-z.

75. Seif, M.; David, G.; Huber, E.; Vallotton, K.; Curt, A.; Freund, P. Cervical Cord Neurodegeneration in Traumatic and NonTraumatic Spinal Cord Injury. J. Neurotrauma 2020, 37, 860-867, doi:10.1089/neu.2019.6694.

76. Wang, K.Y.; Idowu, O.; Thompson, C.B.; Orman, G.; Myers, C.; Riley, L.H.; Carrino, J.A.; Flammang, A.; Gilson, W.; Sadowsky, C.L.; et al. Tract-Specific Diffusion Tensor Imaging in Cervical Spondylotic Myelopathy Before and After Decompressive Spinal Surgery: Preliminary Results. Clin. Neuroradiol. 2017, 27, 61-69, doi:10.1007/s00062-015-0418-7.

Rajasekaran, S.; Yerramshetty, J.S.; Chittode, V.S.; Kanna, R.M.; Balamurali, G.; Shetty, A.P. The Assessment of Neuronal Status in Normal and Cervical Spondylotic Myelopathy Using Diffusion Tensor Imaging. Spine (Phila. Pa. 1976). 2014, 39, 1183-1189, doi:10.1097/BRS.0000000000000369.

78. Zhang, M.; Ou-Yang, H.; Liu, J.; Jin, D.; Wang, C.; Zhang, X.; Zhao, Q.; Liu, X.; Liu, Z.; Lang, N.; et al. Utility of Advanced 
DWI in the Detection of Spinal Cord Microstructural Alterations and Assessment of Neurologic Function in Cervical Spondylotic Myelopathy Patients. J. Magn. Reson. Imaging 2022, 55, 930-940, doi:10.1002/jmri.27894.

79. Mamata, H.; Jolesz, F.A.; Maier, S.E. Apparent diffusion coefficient and fractional anisotropy in spinal cord: Age and cervical spondylosis-related changes. J. Magn. Reson. Imaging 2005, 22, 38-43, doi:10.1002/jmri.20357.

80. Rao, A.; Soliman, H.; Kaushal, M.; Motovylyak, O.; Vedantam, A.; Budde, M.D.; Schmit, B.; Wang, M.; Kurpad, S.N. Diffusion Tensor Imaging in a Large Longitudinal Series of Patients With Cervical Spondylotic Myelopathy Correlated With LongTerm Functional Outcome. Neurosurgery 2018, 83, 753-760, doi:10.1093/neuros/nyx558.

81. Mair, W.G.P.; Druckman, R. The pathology of spinal cord lesions and their relation to the clinical features in protrusion of cervical intervertebral discs (a report of four cases). Brain 1953, 76, 70-91, doi:10.1093/brain/76.1.70.

82. Lindberg, P.G.; Sanchez, K.; Ozcan, F.; Rannou, F.; Poiraudeau, S.; Feydy, A.; Maier, M.A. Correlation of force control with regional spinal DTI in patients with cervical spondylosis without signs of spinal cord injury on conventional MRI. Eur. Radiol. 2016, 26, 733-742, doi:10.1007/s00330-015-3876-z.

83. Cohen-Adad, J.; Alonso-Ortiz, E.; Abramovic, M.; Arneitz, C.; Atcheson, N.; Barlow, L.; Barry, R.L.; Barth, M.; Battiston, M.; Büchel, C.; et al. Generic acquisition protocol for quantitative MRI of the spinal cord. Nat. Protoc. 2021, 16, 4611-4632, doi:10.1038/s41596-021-00588-0.

84. Tuch, D.S.; Reese, T.G.; Wiegell, M.R.; Makris, N.; Belliveau, J.W.; Van Wedeen, J. High angular resolution diffusion imaging reveals intravoxel white matter fiber heterogeneity. Magn. Reson. Med. 2002, 48, 577-582, doi:10.1002/mrm.10268.

85. Caruyer, E.; Lenglet, C.; Sapiro, G.; Deriche, R. Design of multishell sampling schemes with uniform coverage in diffusion MRI. Magn. Reson. Med. 2013, 69, 1534-1540, doi:10.1002/mrm.24736.

86. Behrens, T.E.J.; Woolrich, M.W.; Jenkinson, M.; Johansen-Berg, H.; Nunes, R.G.; Clare, S.; Matthews, P.M.; Brady, J.M.; Smith, S.M. Characterization and Propagation of Uncertainty in Diffusion-Weighted MR Imaging. Magn. Reson. Med. 2003, 50, 10771088, doi:10.1002/mrm.10609.

87. Panagiotaki, E.; Schneider, T.; Siow, B.; Hall, M.G.; Lythgoe, M.F.; Alexander, D.C. Compartment models of the diffusion MR signal in brain white matter: A taxonomy and comparison. Neuroimage 2012, 59, 2241-2254, doi:10.1016/j.neuroimage.2011.09.081.

88. Zhang, H.; Schneider, T.; Wheeler-Kingshott, C.A.; Alexander, D.C. NODDI: Practical in vivo neurite orientation dispersion and density imaging of the human brain. Neuroimage 2012, 61, 1000-1016, doi:10.1016/j.neuroimage.2012.03.072.

89. Le Bihan, D. What can we see with IVIM MRI? Neuroimage 2019, 187, 56-67, doi:10.1016/j.neuroimage.2017.12.062.

90. Lévy, S.; Rapacchi, S.; Massire, A.; Troalen, T.; Feiweier, T.; Guye, M.; Callot, V. Intravoxel Incoherent Motion at 7 Tesla to quantify human spinal cord perfusion: limitations and promises. Magn. Reson. Med. 2020, 84, 1198-1217, doi:10.1002/mrm.28195.

91. Lévy, S.; Freund, P.; Callot, V.; Seif, M. Spinal cord perfusion mapping using Intra-Voxel Incoherent Motion at 3T in healthy individuals and Degenerative Cervical Myelopathy patients. Proc. 29th Annu. Meet. ISMRM 2021, doi:10.1002/mrm.28195.

92. Helms, G.; Dathe, H.; Kallenberg, K.; Dechent, P. High-resolution maps of magnetization transfer with inherent correction for RF inhomogeneity and T1 relaxation obtained from 3D FLASH MRI. Magn. Reson. Med. 2008, 60, 1396-1407, doi:10.1002/mrm.21732.

93. Schmierer, K.; Scaravilli, F.; Altmann, D.R.; Barker, G.J.; Miller, D.H. Magnetization transfer ratio and myelin in postmortem multiple sclerosis brain. Ann. Neurol. 2004, 56, 407-415, doi:10.1002/ana.20202.

94. Fatemi, A.; Smith, S.A.; Dubey, P.; Zackowski, K.M.; Bastian, A.J.; Van Zijl, P.C.; Moser, H.W.; Raymond, G. V.; Golay, X. Magnetization transfer MRI demonstrates spinal cord abnormalities in adrenomyeloneuropathy. Neurology 2005, 64, 17391745, doi:10.1212/01.WNL.0000164458.02141.06.

95. Paliwal, M.; Weber, K.A.; Hopkins, B.S.; Cantrell, D.R.; Hoggarth, M.A.; Elliott, J.M.; Dahdaleh, N.S.; Mackey, S.; Parrish, T.D.; Dhaher, Y.; et al. Magnetization Transfer Ratio and Morphometrics of the Spinal Cord Associates with Surgical 
Recovery in Patients with Degenerative Cervical Myelopathy. World Neurosurg. 2020, 144, e939-e947, doi:10.1016/j.wneu.2020.09.148.

96. Öz, G.; Alger, J.R.; Barker, P.B.; Bartha, R.; Bizzi, A.; Boesch, C.; Bolan, P.J.; Brindle, K.M.; Cudalbu, C.; Dinçer, A.; et al. Clinical Proton MR Spectroscopy in Central Nervous System Disorders. Radiology 2014, 270, 658-679, doi:10.1148/radiol.13130531.

97. Öz, G. Imaging Neurodegeneration: What Can Magnetic Resonance Spectroscopy Contribute? In Contemporary Clinical Neuroscience; Springer, Cham, 2016; pp. 1-11.

98. Joers, J.M.; Adanyeguh, I.M.; Deelchand, D.K.; Hutter, D.H.; Eberly, L.E.; Iltis, I.; Bushara, K.O.; Lenglet, C.; Henry, P.-G.; Professor, A. Spinal cord MRI and MRS Detect Early-stage Alterations and Disease Progression in Friedreich Ataxia. medRxiv 2022, 2022.01.28.22270048, doi:10.1101/2022.01.28.22270048.

99. Aleksanderek, I.; McGregor, S.M.K.; Stevens, T.K.; Goncalves, S.; Bartha, R.; Duggal, N. Cervical spondylotic myelopathy: Metabolite changes in the primary motor cortex after surgery. Radiology 2017, 282, 817-825, doi:10.1148/radiol.2016152083.

100. Holly, L.T.; Ellingson, B.M.; Salamon, N. Metabolic imaging using proton magnetic spectroscopy as a predictor of outcome after surgery for cervical spondylotic myelopathy. Clin. Spine Surg. 2017, 30, E615-E619, doi:10.1097/BSD.0000000000000248.

101. Ellingson, B.M.; Salamon, N.; Hardy, A.J.; Holly, L.T. Prediction of neurological impairment in cervical spondylotic myelopathy using a combination of diffusion mri and proton $\mathrm{mr}$ spectroscopy. PLoS One 2015, 10, e0139451, doi:10.1371/journal.pone.0139451.

102. Aleksanderek, I.; Stevens, T.K.; Goncalves, S.; Bartha, R.; Duggal, N. Metabolite and functional profile of patients with cervical spondylotic myelopathy. J. Neurosurg. Spine 2017, 26, 547-553, doi:10.3171/2016.9.SPINE151507.

103. Salamon, N.; Ellingson, B.M.; Nagarajan, R.; Gebara, N.; Thomas, A.; Holly, L.T. Proton magnetic resonance spectroscopy of human cervical spondylosis at 3T. Spinal Cord 2013, 51, 558-563, doi:10.1038/sc.2013.31.

104. Holly, L.T.; Freitas, B.; McArthur, D.D.L.; Salamon, N. Proton magnetic resonance spectroscopy to evaluate spinal cord axonal injury in cervical spondylotic myelopathy: Laboratory investigation. J. Neurosurg. Spine 2009, 10, 194-200, doi:10.3171/2008.12.SPINE08367.

105. Wilson, M.; Andronesi, O.; Barker, P.B.; Bartha, R.; Bizzi, A.; Bolan, P.J.; Brindle, K.M.; Choi, I.; Cudalbu, C.; Dydak, U.; et al. Methodological consensus on clinical proton MRS of the brain: Review and recommendations. Magn. Reson. Med. 2019, 82, 527-550, doi:10.1002/mrm.27742.

106. Wyss, P.O.; Huber, E.; Curt, A.; Kollias, S.; Freund, P.; Henning, A. MR spectroscopy of the cervical spinal cord in chronic spinal cord injury. Radiology 2019, 291, 131-138, doi:10.1148/radiol.2018181037.

107. Deelchand, D.K.; Ho, M.-L.; Nestrasil, I. Ultra-High-Field Imaging of the Pediatric Brain and Spinal Cord. Magn. Reson. Imaging Clin. N. Am. 2021, 29, 643-653, doi:10.1016/j.mric.2021.06.013.

108. Roussel, T.; Le Fur, Y.; Guye, M.; Viout, P.; Ranjeva, J.; Callot, V. Respiratory-triggered quantitative MR spectroscopy of the human cervical spinal cord at 7 T. Magn. Reson. Med. 2022, doi:10.1002/mrm.29182.

109. Juchem, C.; Cudalbu, C.; Graaf, R.A.; Gruetter, R.; Henning, A.; Hetherington, H.P.; Boer, V.O. B 0 shimming for in vivo magnetic resonance spectroscopy: Experts' consensus recommendations. NMR Biomed. 2021, 34, e4350, doi:10.1002/nbm.4350.

110. Andronesi, O.C.; Bhattacharyya, P.K.; Bogner, W.; Choi, I.; Hess, A.T.; Lee, P.; Meintjes, E.M.; Tisdall, M.D.; Zaitsev, M.; Kouwe, A. Motion correction methods for MRS: experts' consensus recommendations. NMR Biomed. 2021, 34, doi:10.1002/nbm.4364.

111. Deelchand, D.K.; Henry, P.; Joers, J.M.; Auerbach, E.J.; Park, Y.W.; Kara, F.; Ratai, E.; Kantarci, K.; Öz, G. Plug-and-play advanced magnetic resonance spectroscopy. Magn. Reson. Med. 2022, doi:10.1002/mrm.29164.

112. Maier, I.L.; Hofer, S.; Eggert, E.; Schregel, K.; Psychogios, M.-N.; Frahm, J.; Bähr, M.; Liman, J. T1 Mapping Quantifies Spinal Cord Compression in Patients With Various Degrees of Cervical Spinal Canal Stenosis. Front. Neurol. 2020, 11, 1-8, doi:10.3389/fneur.2020.574604. 
113. Baucher, G.; Rasoanandrianina, H.; Levy, S.; Pini, L.; Troude, L.; Roche, P.-H.; Callot, V. T1 Mapping for Microstructural Assessment of the Cervical Spinal Cord in the Evaluation of Patients with Degenerative Cervical Myelopathy. Am. J. Neuroradiol. 2021, 42, 1348-1357, doi:10.3174/ajnr.A7157.

114. Liu, H.; MacMillian, E.L.; Jutzeler, C.R.; Ljungberg, E.; MacKay, A.L.; Kolind, S.H.; Mädler, B.; Li, D.K.B.; Dvorak, M.F.; Curt, A.; et al. Assessing structure and function of myelin in cervical spondylotic myelopathy. Neurology 2017, 89, 602-610, doi:10.1212/WNL.0000000000004197.

115. Dvorak, A. V.; Ljungberg, E.; Vavasour, I.M.; Lee, L.E.; Abel, S.; Li, D.K.B.; Traboulsee, A.; MacKay, A.L.; Kolind, S.H. Comparison of multi echo T2 relaxation and steady state approaches for myelin imaging in the central nervous system. Sci. Rep. 2021, 11, 1369, doi:10.1038/s41598-020-80585-7.

116. Glover, G.H. Overview of Functional Magnetic Resonance Imaging. Neurosurg. Clin. N. Am. 2011, 22, 133-139, doi:10.1016/j.nec.2010.11.001.

117. Duggal, N.; Rabin, D.; Bartha, R.; Barry, R.L.; Gati, J.S.; Kowalczyk, I.; Fink, M. Brain reorganization in patients with spinal cord compression evaluated using fMRI. Neurology 2010, 74, 1048-1054, doi:10.1212/WNL.0b013e3181d6b0ea.

118. Tan, Y.; Zhou, F.; Wu, L.; Liu, Z.; Zeng, X.; Gong, H.; He, L. Alteration of Regional Homogeneity within the Sensorimotor Network after Spinal Cord Decompression in Cervical Spondylotic Myelopathy: A Resting-State fMRI Study. Biomed Res. Int. 2015, 2015, 1-6, doi:10.1155/2015/647958.

119. Cronin, A.E.; Detombe, S.A.; Duggal, C.A.; Duggal, N.; Bartha, R. Spinal cord compression is associated with brain plasticity in degenerative cervical myelopathy. Brain Commun. 2021, 3, 1-12, doi:10.1093/braincomms/fcab131.

120. Hrabálek, L.; Hok, P.; Hluštík, P.; Čecháková, E.; Wanek, T.; Otruba, P.; Vaverka, M.; Kaňovský, P. Longitudinal brain activation changes related to electrophysiological findings in patients with cervical spondylotic myelopathy before and after spinal cord decompression: an fMRI study. Acta Neurochir. (Wien). 2018, 160, 923-932, doi:10.1007/s00701-018-3520-1.

121. Liu, X.; Qian, W.; Jin, R.; Li, X.; Luk, K.D.; Wu, E.X.; Hu, Y. Amplitude of Low Frequency Fluctuation (ALFF) in the Cervical Spinal Cord with Stenosis: A Resting State fMRI Study. PLoS One 2016, 11, e0167279, doi:10.1371/journal.pone.0167279.

122. Powers, J.; Ioachim, G.; Stroman, P. Ten Key Insights into the Use of Spinal Cord fMRI. Brain Sci. 2018, 8, 173, doi:10.3390/brainsci8090173.

123. Eippert, F.; Kong, Y.; Jenkinson, M.; Tracey, I.; Brooks, J.C.W. Denoising spinal cord fMRI data: Approaches to acquisition and analysis. Neuroimage 2017, 154, 255-266, doi:10.1016/j.neuroimage.2016.09.065.

124. Ellingson, B.M.; Woodworth, D.C.; Leu, K.; Salamon, N.; Holly, L.T. Spinal Cord Perfusion MR Imaging Implicates Both Ischemia and Hypoxia in the Pathogenesis of Cervical Spondylosis. World Neurosurg. 2019, 128, e773-e781, doi:10.1016/j.wneu.2019.04.253.

125. UEMURA, K.; MATSUMURA, A.; ISOBE, T.; ANNO, I.; KAWAMURA, H.; MINAMI, M.; TSUKADA, A. PerfusionWeighted Magnetic Resonance Imaging of the Spinal Cord in Cervical Spondylotic Myelopathy. Neurol. Med. Chir. (Tokyo). 2006, 46, 581-588, doi:10.2176/nmc.46.581.

126. Zhou, F.; Huang, M.; Wu, L.; Tan, Y.; Guo, J.; Zhang, Y.; He, L.; Gong, H. Altered perfusion of the sensorimotor cortex in patients with cervical spondylotic myelopathy: an arterial spin labeling study. J. Pain Res. 2018, Volume 11, 181-190, doi:10.2147/JPR.S148076.

127. Sherman, J.L.; Nassaux, P.Y.; Citrin, C.M. Measurements of the normal cervical spinal cord on MR imaging. Am. J. Neuroradiol. 1990, 11, 369-372.

128. Cohen-Adad, J.; Alonso-Ortiz, E.; Abramovic, M.; Arneitz, C.; Atcheson, N.; Barlow, L.; Barry, R.L.; Barth, M.; Battiston, M.; Büchel, C.; et al. Open-access quantitative MRI data of the spinal cord and reproducibility across participants, sites and manufacturers. Sci. Data 2021, 8, 219, doi:10.1038/s41597-021-00941-8.

129. Samson, R.S.; Lévy, S.; Schneider, T.; Smith, A.K.; Smith, S.A.; Cohen-Adad, J.; Gandini Wheeler-Kingshott, C.A.M. ZOOM or Non-ZOOM? Assessing Spinal Cord Diffusion Tensor Imaging Protocols for Multi-Centre Studies. PLoS One 2016, 11, 
e0155557, doi:10.1371/journal.pone.0155557.

130. Andersson, J.L.R.; Skare, S.; Ashburner, J. How to correct susceptibility distortions in spin-echo echo-planar images: application to diffusion tensor imaging. Neuroimage 2003, 20, 870-888, doi:10.1016/S1053-8119(03)00336-7.

131. Andersson, J.L.R.; Sotiropoulos, S.N. An integrated approach to correction for off-resonance effects and subject movement in diffusion MR imaging. Neuroimage 2016, 125, 1063-1078, doi:10.1016/j.neuroimage.2015.10.019.

132. De Leener, B.; Fonov, V.S.; Collins, D.L.; Callot, V.; Stikov, N.; Cohen-Adad, J. PAM50: Unbiased multimodal template of the brainstem and spinal cord aligned with the ICBM152 space. Neuroimage 2018, 165, 170-179, doi:10.1016/j.neuroimage.2017.10.041.

133. De Leener, B.; Lévy, S.; Dupont, S.M.; Fonov, V.S.; Stikov, N.; Louis Collins, D.; Callot, V.; Cohen-Adad, J. SCT: Spinal Cord Toolbox, an open-source software for processing spinal cord MRI data. Neuroimage 2017, 145, 24-43, doi:10.1016/j.neuroimage.2016.10.009.

134. McLachlin, S.; Leung, J.; Sivan, V.; Quirion, P.; Wilkie, P.; Cohen-Adad, J.; Whyne, C.M.; Hardisty, M.R. Spatial correspondence of spinal cord white matter tracts using diffusion tensor imaging, fibre tractography, and atlas-based segmentation. Neuroradiology 2021, 63, 373-380, doi:10.1007/s00234-021-02635-9.

135. Dostál, M.; Keřkovský, M.; Staffa, E.; Bednařík, J.; Šprláková-Puková, A.; Mechl, M. Voxelwise analysis of diffusion MRI of cervical spinal cord using tract-based spatial statistics. Magn. Reson. Imaging 2020, 73, 23-30, doi:10.1016/j.mri.2020.07.008.

136. Gros, C.; De Leener, B.; Badji, A.; Maranzano, J.; Eden, D.; Dupont, S.M.; Talbott, J.; Zhuoquiong, R.; Liu, Y.; Granberg, T.; et al. Automatic segmentation of the spinal cord and intramedullary multiple sclerosis lesions with convolutional neural networks. Neuroimage 2019, 184, 901-915, doi:10.1016/j.neuroimage.2018.09.081.

137. Perone, C.S.; Calabrese, E.; Cohen-Adad, J. Spinal cord gray matter segmentation using deep dilated convolutions. Sci. Rep. 2018, 8, 5966, doi:10.1038/s41598-018-24304-3.

138. Jenkinson, M.; Beckmann, C.F.; Behrens, T.E.J.; Woolrich, M.W.; Smith, S.M. FSL. Neuroimage 2012, 62, 782-790, doi:10.1016/j.neuroimage.2011.09.015.

139. Penny, W.; Friston, K.; Ashburner, J.; Kiebel, S.; Nichols, T. Statistical Parametric Mapping; Elsevier, 2007 ; ISBN 9780123725608.

140. Garyfallidis, E.; Brett, M.; Amirbekian, B.; Rokem, A.; van der Walt, S.; Descoteaux, M.; Nimmo-Smith, I. Dipy, a library for the analysis of diffusion MRI data. Front. Neuroinform. 2014, 8, doi:10.3389/fninf.2014.00008.

141. Provencher, S.W. Estimation of metabolite concentrations from localized in vivo proton NMR spectra. Magn. Reson. Med. 1993, 30, 672-679, doi:10.1002/MRM.1910300604.

142. Li, X.; Morgan, P.S.; Ashburner, J.; Smith, J.; Rorden, C. The first step for neuroimaging data analysis: DICOM to NIfTI conversion. J. Neurosci. Methods 2016, 264, 47-56, doi:10.1016/j.jneumeth.2016.03.001.

143. Tetreault, L.; Kopjar, B.; Nouri, A.; Arnold, P.; Barbagallo, G.; Bartels, R.; Qiang, Z.; Singh, A.; Zileli, M.; Vaccaro, A.; et al. The modified Japanese Orthopaedic Association scale: establishing criteria for mild, moderate and severe impairment in patients with degenerative cervical myelopathy. Eur. Spine J. 2017, 26, 78-84, doi:10.1007/s00586-016-4660-8.

144. Roberts, T.T.; Leonard, G.R.; Cepela, D.J. Classifications In Brief: American Spinal Injury Association (ASIA) Impairment Scale. Clin. Orthop. Relat. Res. 2017, 475, 1499-1504, doi:10.1007/s11999-016-5133-4.

145. Jutzeler, C.R.; Ulrich, A.; Huber, B.; Rosner, J.; Kramer, J.L.K.; Curt, A. Improved Diagnosis of Cervical Spondylotic Myelopathy with Contact Heat Evoked Potentials. J. Neurotrauma 2017, 34, 2045-2053, doi:10.1089/neu.2016.4891. 